\title{
Further experimental investigations on variation of types, and on the nature of quantitative differences in Daphnia types.
}

\author{
Richard Woltereck \\ Contact: maximilian@alumni.reed.edu)
}

\begin{abstract}
The following is a translation from the German of Richard Woltereck's classic paper ${ }^{1}$ introducing the idea of reaction norms; a concept which encapsulated the now-ubiquitous idea of a genotype-by-environment interaction, and contributed to the idea of the developmental program.

Woltereck begins with a brief discussion of Lamarckist, mutationist, and selectionist views on heritability, the influence of the environment (he uses the German term "Milieu"), and variation of "types". (When Woltereck writes about such types, he is referring specifically to idea of pure or true-breeding lines arising from Johannsen's work under the mutationist model.) This is followed by a discussion of methods for understanding these concepts in biological systems. A research plan is outlined for distinguishing between these possibilities in a fashion to be generally convincing among the diverse factions. Data following the initial steps of this research plan are presented. An introduction to the model system of Daphnia is provided, and in particular the interesting response of head morphology to seasonal variation ("cyclomorphosis").

The basic observation of consistent responses to the environment among clonal Daphnia is presented and analyzed in light of both temperature and nutritional variation, and in terms of physiology and morphogenesis. Woltereck goes on to determine that manipulating food quantity is sufficient to recapitulate this variation in the laboratory. He calls the response of a genotype to environmental variation a reaction norm.

Woltereck develops his theme by comparing different genotypes (captured under Johannsen's terms of "Biotype" and 'elementary type", or simply "type") in their reactions to different environmental conditions. He further contrasts the observed data to the expectations under Johannsen's simple expectations of the independence (meaning here additivity) of contributions of genotype and environment to mean values of phenotypes. He finds that this expectation of independence is
\end{abstract}

Copyright (c) 2020, Maximilian Oliver Press. All rights reserved.

${ }^{1}$ Translation by Maximilian O. Press. The text is drawn from pages 110-173 of the Verhandlungen der Deutschen Zoologischen Gesellschaft, vol. 19, 1909, as digitized by the Biodiversity Heritage Library (urlhttp://www.biodiversitylibrary.org/item/182001). I copyrighted the translation under my own name for lack of a better idea. not borne out in the data. Moreover, the differences between environments varies from negligible to dramatic even for the same genotypes, and thus the differences do not comport well with a discontinuous model of mutational variation. Instead, the differences between types are continuous and difficult to predict from bulk genotypic or environmental differences.

Woltereck then shows that similar dynamics underlie variation of sexuality frequency differences in Daphnia genotypes, indicating that his results are not a simply a peculiarity of the head-height character.

In the paper's third section, Woltereck examines some apparently discontinuous characters related to the presence/absence of two different atavistic morphological structures, the nebenaugen and the crown toothlet. He concludes that they are in fact continuous characters. furthermore, he finds that these stochastically-appearing characters can sometimes be heritable, but that their appear to have at least some environmental basis. Furthermore, they respond to selection in a way that suggests genetic assimilation sensu Waddington.

The paper finishes with some preliminary experiments (reported more fully in a later 1911 paper) by which Woltereck aims to generate heritable quantitative variation in the reaction norm, which is to say the genotype, through sustained environmental exposure. Specifically, he aims to enforce a certain above-average head-height as a fixed genotypically-specified character through the environmental conditions that lead to tall heads in the ancestral reaction norm. The aim is to alter the reaction norm such that these tall heads appear even without the driving environmental stimulus. Here again it appears that he has anticipated and clearly described what Waddington later called genetic assimilation. Woltereck demonstrates the gradual stabilization of head height over the course of years.

Woltereck's paper finishes with four appendices discussing certain topics in greater detail, including a hypothetical mechanism for what we now call genetic assimilation. It is followed by brief discussions from two other researchers, whose style of review will be familiar to many present-day workers.

For a brief review discussing these results in the context of the time, I direct the reader's attention to my translation of Keilhack 1909. Throughout I have occasionally included footnotes drawing attention to pertinent sections; these are in italic face and prefixed with $M O P$ to distinguish them from Woltereck's own notes. I fo- 
cus these in places on Woltereck's comprehension of Johannsen's genotype-phenotype division, and his apparent discovery of what we now might call genetic assimilation.
Outline Preface: General. -- Necessary Materials. -- Isolation of the Elementary Types. - Plan of Investigation. Observations on the Crossing and Selection of Daphnia.

1. Ascertainment and analysis of the quantitative character "head-height".

(a) The basis of different head-heights.

(b) Assessment and description of the collected phenotypes.

(c) Genotypical basis of head-height and different genotypes.

i. Genotype $=$ reaction norm

ii. The beginnings of genotypical distinction - Natural Transitions.

iii. Artificial transitions

iv. Differences of reaction of generations

2. Analysis of the phenotypic and genotypic differences of the Quantitative character "sexuality"

(a) Exact determination and quantitative measurement; causes of the differences

(b) The beginnings of genotypical distinction - Natural Transitions.

(c) Artificial transitions

(d) Differences of reaction of generations

i. Specific generation sequences

ii. Their relevance to natural environments

3. Undertakings and experiments concerning regressive variation

4. Experiments towards generating new genotypical quantitative characters through the influence of the environment

Appendices:

1. The causes of continuous variation in Johannsen's experiments and my own

2. On the metabolic physiology of Cladocerans

3. Examples of partial alterations of the reaction norm

4. Sexuality and generational cycles

\section{Preface}

From the results of many experiments, in particular botanical studies of inheritance of the last century-on the one hand the Mendel's crosses, on the other the pure breeding experiments of Johannsen, and most lately the famous mutations of de Vries-we have derived a new idea regarding the variation of types. This concept, which is championed especially by de Vries and W. Johannsen ${ }^{2}$, stands in nearirreconcilable opposition to the Lamarckist inclinations of

\footnotetext{
${ }^{2}$ Compare especially Johannsen's new book: Elemente der exakten Erblichkeitslehre. Jena, G. Fischer, 1909 (515 p.).

See also the report of Arnold Lang in this journal. Finally I would like to draw attention to the important work of the Heidelberg botanist Georg Klebs, without which I could not have completed the present work. Klebs has demonstrated the exceptional scope of continuous, environment-dependent variability in several plants (Sempervivum, Sedum). See also his "Studien über Variation" (Archivf. Entwicklungsmech. 1907)
} 
today's Botany, and also of most zoologists, regardless of whether they consider themselves Lamarckists or Selectionists.

We hear the following: the elementary types (not concerning ourselves with the artificial umbrella term of systematic "species") differ from each other through the assortment of predispositions, determinants, or genes, which are inherited in their germplasm. So far the "exact doctrine of heredity" (Johannsen) corresponds to the older teachings, especially Weismann. Moreover, this doctrine provides the most striking evidence for the existence and continuity of the germplasm-elements that Weismann could wish for, with regard to the correctness of his theoretical ideas. The dissent of the new teachings concerns only the variation inherent in these elements:

1. These hereditary elements are of fixed quantities, which do not show the smallest variability in siblings (respective in germplasm to their parents), or show any gradual variation across many generations. A change in these elements, and thereby the heritable characters of the elementary type, follows exclusively through spontaneous mutations; fluctuating, individual, or continuous variability is negligible with respect to the heritable nature of types. ${ }^{3}$

2. The nature of change of the hereditary elements, and therefore the direction of these mutations, does not have a causal relationship to the local conditions, or environment. Extreme changes of environment may prefer the emergence of certain mutations, and thereby "disturb the equilibrium of the germplasm", but the variation of germplasm and type thus unleashed does not indicate any causal relationship of the mutation to the environment.

Therefore, the environment in no way determines the direct inheritance of type variations (Lamarckism), and even its indirect action (harvesting the most adequate variants; that is, selectionism) is secondary. Selection does not decree the multitudes of available, fluctuating germ-variants (and absolutely cannot impart a direction on the organs, tissues, or germ cells). Its scope is restricted to the final mutations, which appear only occasionally. Here it will eradicate the unworkable mutants through the preference for certain forms, at least so far as they have not already left the stage through inviability or sterility.

Therefore: there is no continuously-arising variation and equally no continuously-arising evolution.

And there is no determining influence of the environment, except through the excision of unfavorable mutants.

These are hard blows against that conception of the development of the organic world in which we are tutored. However, the zoologists present so many illuminating examples of finely-graduated arrays of forms and adaptations that it is difficult to accept a conception of discontinuous development. By these little steps of variation in types the influence of the environment (whether direct or indirect) can be explained, without giving up our lone mental anchor to an unmetaphysical understanding of organic development.

\footnotetext{
${ }^{3}$ translator's note: these terms are taken from De Vries' writings in The Mutation Theory, and the vocabulary is shared by Johannsen.
}

However, the advocates of the "exact doctrine of heredity" support themselves with analytical experiments, and therefore they see all the rich body of facts that have accumulated since Darwin as inconsequential-dashing our hopes, like a tanner who loses his hides ${ }^{4}$. Of course: experimental analysis is that rich goal towards which observational facts are only the smallest steps.

Therefore, if we want to meaningfully fight against the new doctrines we must use the weapons of the enemy, meaning principally analytical experiments. Then we will compare the worth the "old" conception: continuous variation of type under a determining environment; to the "new": discontinuous variation without a determining environment. It must be clear that up to this point only the new view has been supported by experimental tests, and not the old.

\section{Necessary Materials for Investigating the Variation of Types.}

If we wish to assess with any precision whether organic properties vary continuously or in jumps, it would be best to make use of predefined quantitative characters (e.g. length measurements). In these characters, variation can be specified numerically and unambiguously. It is, in my opinion, a weakness in Johannsen's new doctrine, that he favors qualitative categorical characters (e.g. color) that are altogether subjective, rather than quantitative characters. ${ }^{5} \mathrm{We}$ must take greater pains to eliminate the influence of crossing and sex in our experiments, such that only sexless, parthenogenetic, or self-fertilizing organisms are appropriate. In this sense, it is indispensable that we study variation and heredity in "pure lines" (Johannsen).

Third, finally, it is preferable that we only have to do with organisms that display a large number of small (but heritable) differences for analysis. The comparative study will then concern itself with forms that show no substantial difference, other than one or a few quantifiable contrasts, which in comparison constitute elementary types ("Biotypes" for Johannsen).

The famous Danish botanist succeeded in cleanly identifying such elementary types in great numbers within landraces of cereals and beans. We require similarly numerous and diverse animals for zoological studies to design a comparable experiment, for instance Daphnia or other Cladocerans that are available in our lakes and ponds. For an account of landraces in Daphnia, I can recommend my own work showing different heritable Biotypes ${ }^{6}$.

Both of the other requirements are fulfilled either in Johannsen's work, or in my Daphnia. Johannsen isolated "clean lines" through self-fertilization, whereas I used parthenogenetic propagation under controlled, constant culture conditions while removing the eggs with ephippia (egg casings generated during sexual reproduction), such that the

\footnotetext{
${ }^{4}$ translator's note: ????

5 translator's note: Johannsen did in fact work on both quantitative and qualitative characters, see for example his 1903 monograph on heritability in populations and pure lines.

${ }^{6}$ Verh. d. D. Zool. Ges. 1908, vol. 18, pp. 234-240
} 


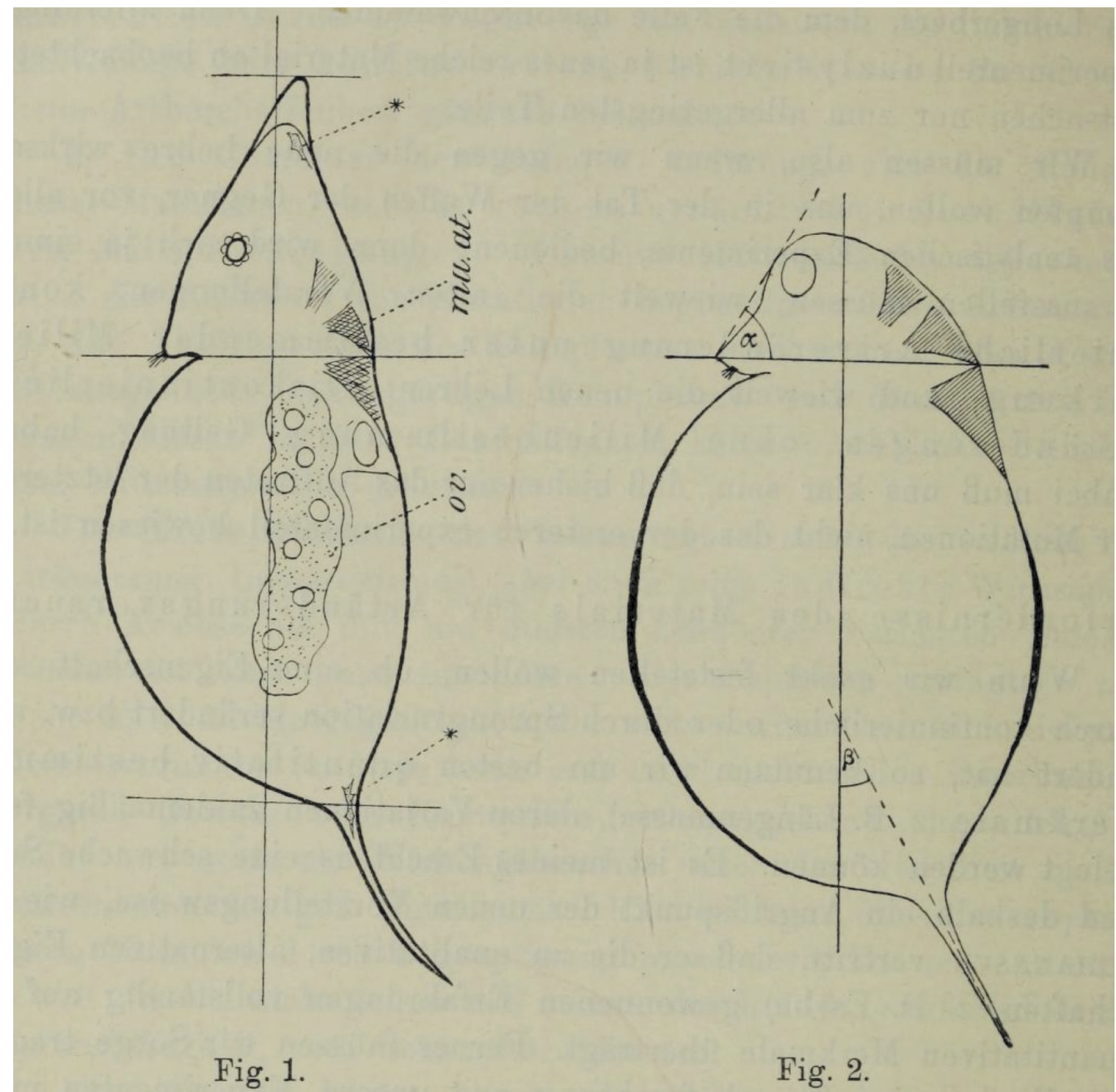

Fig. 1. Side view of Hyalodaphnia cucullata-o of intermediate height. The superimposed axes allow the determination of head height. One obtains a single useful "head-base" by drawing a line connecting the point of the rostrum (bottom edge) to the border between the second and third antenna-muscles (indicated $m u$. at). The midpoint of this line yields a perpendicular line describing the body's long axis, along which we can measure head height and the length of the carapace as shown. Out of these individual measurements one can compute the "relative head height", expressed as a percentage of carapace length. It is necessary each time to draw from a photograph, in order to quickly and accurately capture the outline (including the head-base) on the slide, while at the same time preserving the life out of water of the animal in question. Fig. 2. Daphnia longispina from the Lunzer Untersee [translator's note: an alpine lake in Austria. Woltereck was director of a research station in Lunz.]. Angles of the axes indicate the facial angle $(\alpha)$ and the spine angle $(\beta)$. 
fertile eggs develop into $q^{7}{ }^{7}$. Thus the correctly-timed isolation and robust feeding of these 우 suffices across many Daphnia races to maintain pure lines (in some cases for this purpose, the sibling 우 without ephippia should be excluded). Almost invariably under favorable conditions, the animals revert from laying more ephippia to making parthenogenetic eggs (subitan eggs), and indeed almost without exception they are $q$ in individuals that arise out of such ("post-ephippial") virgin eggs. The appearance of $\sigma^{7} \sigma^{\prime}$ in unattended cultures can be dismissed, so long as no ephippia appear; to this end, new generations were obtained by descent through single 우, to entirely exclude this possibility.

As is well known, Johannsen used as quantitative features the length of beans and the serration of barley varieties. In Daphnia, the length of the carapace spines, the height of the head, the facial angle, the spine angle (compare figures 1 and 2), and sexuality (see below) can serve as quantitative characters.

Thus, the materials which Johannsen used and my own Daphnia populations are sufficiently comparable in these three critical points, in spite of the totally unrelated morphologies of higher plants and metazoans.

\section{Isolation of the Elementary Types}

As is well known, Johannsen proceeded from the premise that he isolated variants comprising different elementary types or Biotypes from a initial mixed (though apparently uniform) population. These types proved themselves through the heritability of coherent, unchanging features. The progeny of each individual, for instance the largest and smallest, show similar means and the same range of variation as their parents. (Johannsen's exciting results were that selection on variations within an elementary type does not reveal any heritability of these variants, specifically if the same conditions are used year-to-year. This result shook the foundations of research of statistical variation and heredity, as practiced by the Galton-Pearson school. Specifically, the meaning of the famous regression laws, which to many were the basis of all exact heredity and speciation research, was called into question by Johannsen's findings.)

With Daphnia, obtaining elementary types is easier on one hand, and one the other more complicated. Easier, because most studied natural populations (inhabiting ponds and lakes), are found to be consistent and homogeneous. In the same body of water, in a pond by Torgau measuring more than a square kilometer, I could find two Biotypes in an apparently homogeneous population of Daphnia longispinagaleota. These types showed not-insignificant differences in their "relative phenotypes" and inheritance. Many bodies of water have yielded similar results, so I assume that it is the same for the Danish populations of Daphnia studied so exhaustively by Wesenburg-Lund.

\footnotetext{
${ }^{7}$ translator's note: Throughout, Woltereck uses Mars and Venus astrological symbols to specify male and female organisms. He follows the practice of using two such symbols to denote a plural. I have kept this notation as a matter of taste.
}

Thus, it is not surprising that in a single lake multiple elementary types of Daphnia longispina and cucullata coexist. Rather, it is unanticipated that within such types, only one apparent biotype exists for each elementary type (or subtype). And therefore we must confront the complicated side of our question (compared with the bean and barley biotypes maintained through selfing), concerning the poorly illuminated basis of the identity of types.

In considering Daphnia types, in which sexual reproduction and diapause (dauer stage) play a greater or lesser role, we would expect that the mixing of local forms is substantial. In reality, we observe essentially no type mixing. In the case of such mixing, we should always encounter inconstant forms of variable descent, rather than the striking constancy which the forms show from year to year. ${ }^{8}$

The mixing of local types with strange biotypes appears much less important than the mixing maintained within some local types at the end of the breeding season. Through this general admixture within individual germplasms, the differences within the local types will be balanced out as generations proceed.

The transition between obligatory sex and total parthenogenesis takes at least a year. With most Daphnia, the lakes show a greater or lesser proportion of parthogenetic 우우, while the remaining individuals go over to the production of dauer (diapause) eggs and $\sigma^{x} \sigma^{x}$.

It would be worthwhile also to make a comparative study of the means of inheritance in varieties with different degrees of sexuality. In particular, it would be good to bring up those populations which have given up sexuality entirely (for instance, Bosmina and Daphnia longispina-hyalina of the great alpine lakes). In the case of these last forms we must expect the population to be split up into more biotypes.

For the task at hand, these considerations are of theoretical interest (in the selection and orientation with respect to the working material), but are not practically relevant. In each of my cultures I deal with single biotypes, because if I have selected a lone $q$, the following generations will necessarily be parthenogenetic. These generations represent "pure lines", like those that Johannsen studied.

Thus, we have an initial working material for the experimental analysis of quantitative characters, whose change (whether continuous or discontinuous, and whether with or without determination from the environment) we desire to study.

\section{Research Plan}

The success of our undertaking requires a systematic approach. it must be carried out in such a way that the local forms compared represent elementary types and not only slight variations of the same type, requiring the following tasks:

1. Analysis of the collected modifications that the various characters of a given biotype can show, which are thus caused by the conditions of the environment. We will thereby understand the various potential forms (the "phenotypes" of Johannsen) of each character on the one hand,

\footnotetext{
${ }^{8}$ see my paper Verhandl. 1908, p. 239.
} 
and on the other define the heritable "genotypic" differences between biotypes, in order that we may compare these biotypes under like environments. To eliminate the various influences on individual cases of embryonic development, we must not compare individual measurements, but rather the averages of a rather large count of age-matched individuals of the same generation and environment.

With that said, this first task of analyzing available biotypes depends upon our ability to focus on a single character or trait (for instance, head-height), in three sub-tasks of increasing difficulty:

(a) Exact determination of the range of variation of the relevant character under all possible conditions of the environment.

(b) Exact determination of those conditions of the environment that elicit individual modifications of the character in question.

(c) Exact determination of the (heritable) differences, which are shown in the collected modifications of the relevant character between different biotypes under the same conditions.

2. After these initial steps, a second area of work constitutes the testing of genotypic differences of elementary types through crossing. This may answer the question of whether the ascertained quantitative variations in characters resolve into intermediate forms, or whether they follow the rules of dominance and segregation (and how they do so).

3. Further, we must observe whether biotypes isolated from clean lines can give rise to extreme variants under extended selection.

4. Finally, we must test whether and how far the continuous action of specific conditions of the environment can achieve the change of a character within a biotype.

My experiments have been carried out at this point over the course of three years and concern themselves more or less with the set of tasks under the first heading above. I have also made a beginning of my attack on the remaining questions, but unlike the work presented here, they have not yet achieved clear and final results. An extensive description of my attempts will appear as soon as possible in book from, together with the data itself, laying out my underlying interpretations and conclusions.

\section{Observations on crossing and selection in Daphnia.}

Concerning points 2 and 3 (but little treated in the above enumeration) I will make a few observations here.

Concerning 2, the attempts at crossing have run into obstacles related to methodological difficulties in obtaining perfect data, specifically in generating a sufficiently large number of progeny to obtain average values of the quantitative traits among hybrids. And indeed, there are difficulties ensuring data from these progeny are equivalent, e.g. the same culture and generation, giving rise to equal males and also perfectly equivalent females. We will therefore see in the following results that, for determination of the genotypic values of the quantitative characters, we must also define the 'grades' of the environment and observe the count of the generation.

The relatively few hybrids that I have obtained from numerous experimental crosses between Daphnia longispinaand cucullata local types show intermediate characters, but an unambiguous evaluation is at this point impossible. I address these points here only to alert other researchers that the inviting crossing-experiments (at least for quantitative characters) will only yield value from an extensive phenotypic and genotypic analysis. Even then, we must ensure the material is adequate to the questions under study (average values, the equivalence of the parent stocks).

Concerning 3, the large majority of my experiments attempting to call forth extreme variants under selection upon pure lines have yielded negative results. This is very similar to the results of Johannsen. On the exceptions from this rule, and on the various experimental series where changes have occurred, I cannot at this point provide much clarity. Therefore, in the following pages I will only touch upon these points so far as I believe will serve future researchers.

I will briefly consider some observed mutations in another context, whose isolation (selection) yields satisfactorily strong heritability (p. 14). But these are not very meaningful in terms of the generation of types in Daphnia.

Now, the important question concerns whether a continuous character can be changed only through continuous selection on variants (those increasing or decreasing it, for instance). I wish to emphasize the necessity of clearly separating the influence of the selection from that of the environment in changing this character. This proved quite difficult in Daphnia; it seems doubtful whether factors of the environment (including embryonic nutrition) can be entirely eliminated in the study of selection. Instead, the most promising studies determine selection factors through, for example, comparing the effects of different environments on unselected populations to individuals who have been selected for strong deviations in the character (with matched ages). The influence of selection can then be inferred from the difference between the simple and the combined methodologies. ${ }^{9}$

However, we have not yet come so far. We will see that only one type-changing environment (out of four total) at this point has very preliminary results concerning the artificial elicitation of new genotypes. On the selection of new variants, consult p. 24.

However, as we will see next, analysis of existing Daphnia genotypes may already have yielded compelling conclusions regarding the nature of the variation of types.

\footnotetext{
${ }^{9}$ translator's note: slightly unclear to me which precisely these methodologies are.
} 


\section{Exact determination and analysis of collected phenotypes of the quantitative character "head-height".}

We understand "phenotype", like Johannsen, to signify the average value of a character under some circumstance, for instance the middling length of a stalk in an evenly watered and manured field of rye. This phenotype can be uniform, if all the plants belong to a single elementary type, or only apparently uniform, when multiple different heritable ("genotypic") elements are planted, in other words in a mixed population. However, in the following work we concern ourselves solely with phenotypes whose singularity can be guaranteed through previous hereditary experiments. To simplify the picture further, we prefer to focus on some particular character, namely the head-height of Daphnia landraces (which we treat as elementary types). Further, we obtain the 'relative' head-height by also considering the length of the carapace (compare Fig. 1 and relevant explanation).

Our first task is now to compute the phenotype of headheight by measuring age-matched samples of 100 examples (우 or $\sigma^{7} \sigma^{7}$ ) from natural populations, from which we obtain a frequency curve and can then compute the desired average (phenotype).

Such a calculation would only have worth if we are able to obtain age-matched animals (that have, for example, gone through a like number of molts), as they might also change their relative head-height over their lifetime. We must confine ourselves in this analysis to animals at a roughly similar adult age, preferably considering only the ofo, for the purposes of constructing our curve. We may nonetheless in many cases obtain multimodal curves, if for example adult ㅇ from different generations are included, which can vary in their head-height (particularly early in the year).

It is therefore not permissible to compare the "natural" frequency curves with curves generated from cultured material; we will never make comparisons including individuals from different generations or age classes.

If we perform a similar experiment in wild animals at different times of year, across many Daphnia, we reliably find quite different head-heights in spring, in summer, and in autumn/winter. Thereby, the frequency-curve and its mean continually move; we obtain a spring curve, a summer curve, et cetera. Or more precisely stated: the curve (and with it, the phenotype) of head-height shifts to the right over time from Spring until September, only to return once more to the left (Fig. 3).

Wesenberg-Lund has described the change of head-types across many Daphnia of the Danish lakes, giving us a characteristic example of cyclomorphosis. Of course, these samples of animals are taken directly from the lakes in question, not from cultures.

These observations are thus very informative, collected as they are across many years, in descriptively characterizing the forms of local races. However, in knowing the particular heritable nature (the genotype) of an elementary type, we cannot do without cultures.

In our cultures, we can artificially regenerate all of the different head-heights that we observe in lakes over the course of a year. We must, of course, take care that our culture conditions ${ }^{10}$ be correct and appropriate. I have accomplished this in various attempts to obtain pure pelagic Hyalodaphnia cucullata.

\section{a. The causes of the different head-heights.}

There are three different principal factors from which the different head-heights in the space of a Biotype are derived (similar to other characteristics of form, such as spine length, spine angle, brow angle, and overall size): feeding, temperature, and generation number.

External factors Until this point the role of temperature as a biological factor was thought to be related to its inverse relationship with water friction (at higher temperatures friction is lower), based on Wo. Oswald's ${ }^{11}$ treatment of the subject. Consequently, planktonic organisms require a higher buoyancy to remain in suspension at higher temperatures than they do at lower temperatures.

The buoyancy of Daphnia bodies is apparently increased by, among other things, the body processes (on one side the head-height dependent "helmet", and on the other side the mantle-spike). It can also be increased by reducing overall body size, which increases relative surface area. The hypothesis that relevant body alterations may be triggered or caused by temperature changes is therefore obvious.

However, my cultures show to the contrary that temperature plays only a secondary role in these alterations. Further, head-height is not altered through changes in internal friction. In coming to this last conclusion, I rely primarily on results from the addition of quince mucilage to the medium, which is better borne by the animals than supplementation with glycerin or salt. Considering that the result was always negative (apart from a definite degeneration of the older cultures in higher concentrations), it would not be profitable to further explore the details of these experiments.

Increases and decreases of the temperature itself are in contrast to these results, as Wo. Ostwald very rightly observes (though he does not interpret them properly). Temperature has a noticeable influence on the head-height, in addition to an effect on overall body size.

We shall consider the latter, simpler alteration first. Body size can in fact be reduced by heat, but only when holding constant a poor to middling diet. In contrast, with more food, body size is absolutely not reduced by heat, but rather considerably increased.

The connection here is obvious: under higher temperatures bodies require a larger amount of food for their higher vital activity (movement, heartbeat, and fertility are very obviously increased). Therefore, they must devote less material to their growth, with only a meager quantity of food at their disposal. In contrast, if food is available in surplus (which is unlikely to be true for pelagic types, which sustain themselves upon any available detritus including Bacteria, et

\footnotetext{
${ }^{10}$ Consult my note on the rearing of Daphnia in the Intern. Rev. d. ges. Hydrobiol. 1908, Volume 61, 871-874.

${ }^{11}$ From his numerous writings on this subject, one is especially relevant: "Exper. Unters. über den Saisonpolymorphismus bei Daphniden" (Arch.f. Entwicklungsmech. 1904).
} 


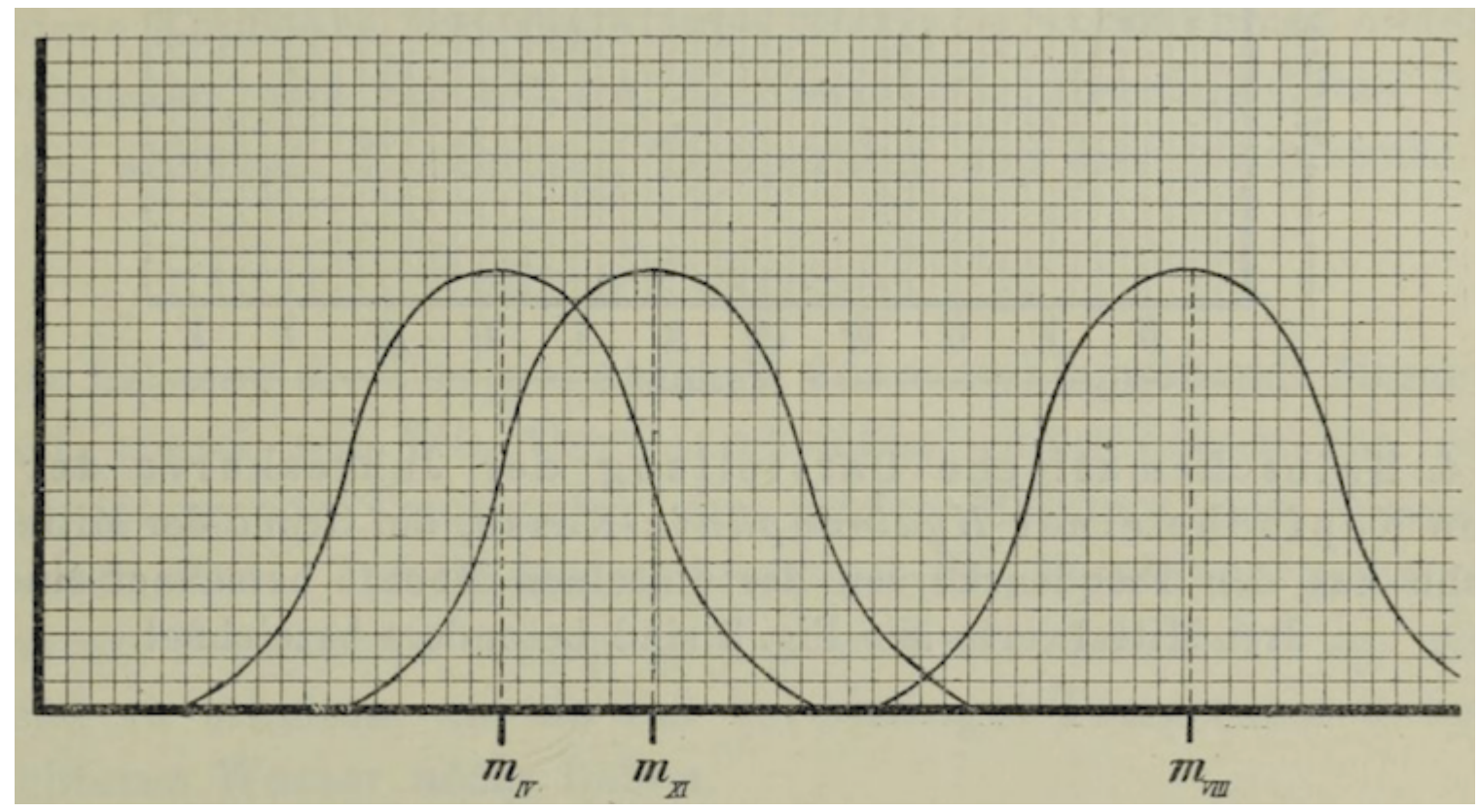

Fig. 3. Semi-schematic representation of three frequency curves of relative head-height of certain Hyalodaphnia races collected from the wild. The horizontal axis represents a measure of relative head-height, and the vertical axis represents the percentage of individuals with a given head-height. The left curve represents grown April-caught o+o , the right August-caught, and the middle November-caught. For each of the three curves, the mean is indicated with $m_{I V}, m_{V I I I}$, and $m_{X I}$, in turn indicating the number of the the month.

cetera), then warm conditions induce an increased capacity for assimilation and with it an increase in body size.

The same principle applies also for head-height or the "Helm" of the animal, as shown in numerous important experiments upon this important point using Daphnia longispina, galeata, and cucullata. I will not go into these experiments in detail, and only share that I have achieved greater head-heights in my cultures with low temperatures and rich diets of Chlorella than I have with high temperature and poor diets. Specifically, after a week-long incubation in a thermostat at $30^{\circ}$, the heads stay small if there is not a rich algae diet (consider that the "natural" temperature ranges from $\left.18-25^{\circ}\right)$. Even at $6-10^{\circ}$, I can achieve higher heads with rich food than I can with lesser nourishment at $30^{\circ}$. At temperatures under $5^{\circ}$ or above about $32^{\circ}$ (the bounds differ between biotypes) metabolism is quite poor, such that past these points animals 'stunt' or die.

The smaller heads that overwintering Daphnia longispina or cucullata show in the colder seasons (Wesenberg-Lund was the first to show that they are all alike) are attributable on the one hand to lesser food, and on the other to a degradation of the assimilation capacity by cold. The high helmets of the summer, however, are achieved through increased food; or more exactly, through the consequently increased assimilation. (Concerning the other innate factors effecting helmet size, compare p. 18 below).

This subject is too complicated to deal with in the space of this article. We can, however, abstract the most essential factors of the 'environment', e.g. the living situation of our Daphnia. It is particularly important that the quantitative differences of assimilation be directly proportional to the quantitative differences of tested characters, while a second trait (sex, p. 19) is held proportionally constant.

It is methodologically important that the influence of temperature, as a secondary factor affecting assimilation intensity, be controlled in analytical experiments. For this, one may simply take care for the warming of the cultures. The same may be said of the total state of the 'environment' of the cultures: salt and gas content, volume, and light in adequate and equal amounts, such that the single factor of food supply is left, and this last is well-measured (see appendix 1, p. 28).

We can thus establish the supplied quantity of food as the measurable cause of head-height particularly. Similarly, we can ignore the quality thereof if we feed comparable cultures with the same pure cultures of Chlorella (or some other algae) in measured amounts. ${ }^{12}$

\footnotetext{
${ }^{12}$ Unfortunately it is often not possible to perform immediately consecutive experiments. This makes it rather uncomfortably noticeable that nutritive value of these algae fluctuates in ways that are difficult to understand (perhaps together with their variable tendency to stay suspended in water or sink to the floor). Especially, care must be taken that food algae must not be grown next to Bacteria or other algae, which often (for instance with the spiked Raphidia) render the algae unpalatable to Daphnia. In such cases one may obtain totally untrustworthy results.
} 


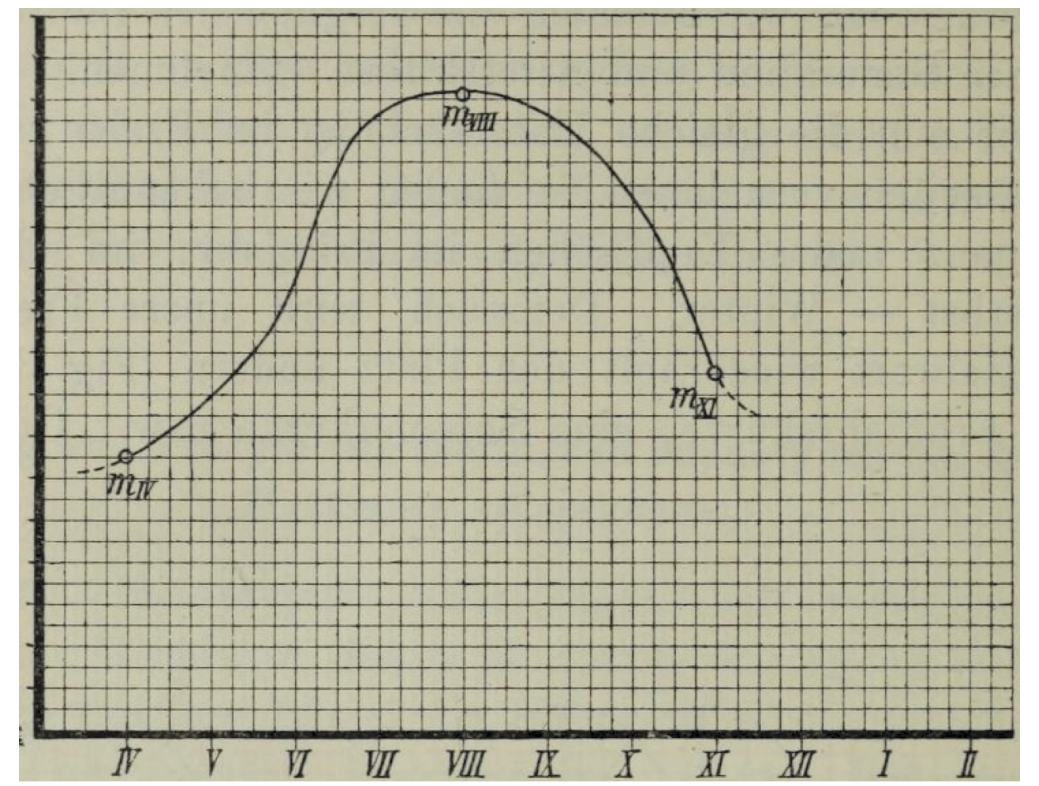

Fig. 4. Semi-schematic representation of the change in relative head-height of a Hyalodaphnia race, based on means calculated from the frequency curve of each month. The vertical axis represents a relative head-height measure, the horizontal access indicates month of the year (as numbered in Roman numerals). Three means from Fig. 3 are indicated.

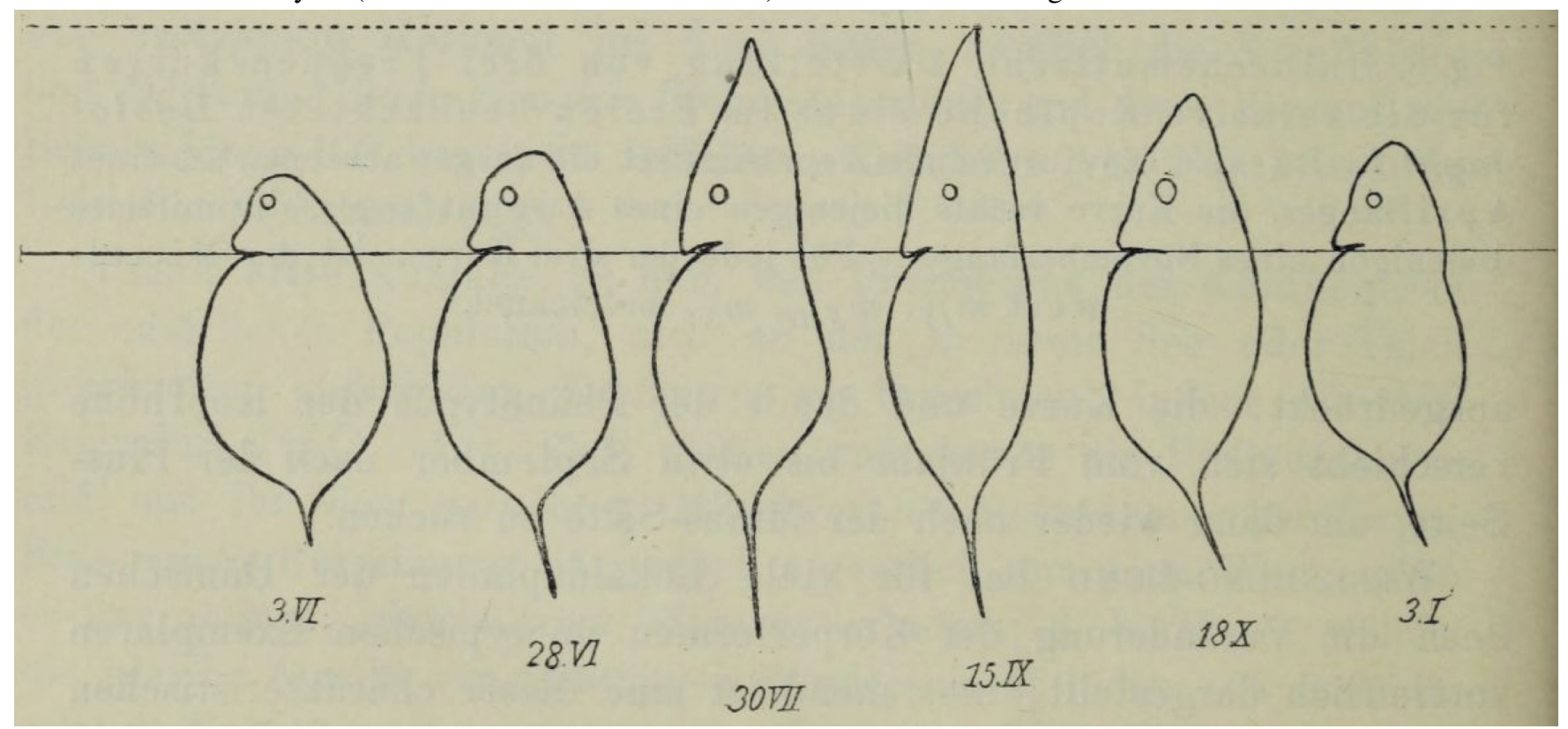

Fig. 5. Representation of the cyclomorphosis of a Hyalodaphnia, after Wesenberg-Lund. These are likely single typical examples of large similar populations, drawn next to each other. (By joining the vertex points one may obtain a year-curve of absolute head-heights, which however is not so useful as the means of relative head-height used in Fig. 4. The examples used by W.-L. for illustration (Plankton-investig. of the Danish Lakes II. 1908) from Skanderborg Lake were chosen because they showed a definite periodicity in both spine-length and spine-angle across months. 
Inner factors We must now put aside nutrition (and the secondary factors of temperature and so on, that we have eliminated through experimental treatments) to take another variable factor into consideration, namely the influence of the generation number. This factor is a part of the residual heritable helmet-potential always at work, whose existence we will shortly observe in detail. We saw above that assimilation intensity (as a product of nutrition and temperature) must be particularly crucial in reshaping both the body plan and the head height. From this we may clearly understand this special assimilation process, and we might say that this operation of nutrition on the helm primordium is directed by inner forces, which are in truth the decisive factor. This is the inherited potential for helm-growth. We can understand this potential as acting on available materials in the hypodermal cells of the head; or better, as an arrangement of these materials, which reacts to the certain qualities and quantities of nutrition (blood) through the construction of a ridge-like anterior process of a certain height.

We now approach what seems to me an especially important point, so it will be beneficial to briefly consider the morphological and physiological components of this helmgrowth. The critical region of the Daphnia head is normally found by comparison to the chitin-covered hypodermis, which is thickened on the rest of the body. When no helm is present, this has something of the form of a roof. Invariably (except in the case of very low heads) this roof shows a sharp ridge; the systematists say that the head is 'keeled'.

The thickened hypodermis underlying this ridge or keel is the seat of the 'helm-potential', whose nature we would like to investigate. The hypodermis can remain low and compact throughout life (as shown by Fig. 6a), it can however also grow out to a high and narrow protrusion (Fig. 6b), which then is covered with a thin layer of chitin and takes on the appearance of a helmet when viewed from the side.

In reality the 'helm' is a narrow blade; the young animals in the mother's brood compartment maintain it, so long as their chitin is not yet hardened. In such close spaces it resembles a sleeping cap turned down to the left or the right.

But how does the stimulus control the helm-forming potential of this organ? This may occur by two quite different ways, depending whether it acts upon the helm-building cells of an embryo or of an adult animal. Namely, in the latter case, this cell mass retains the potential for development throughout life (or at least until the 'advanced age' of 10-12 weeks). Of course, in younger animals the reaction proceeds much faster and more completely than in older examples.

In embryos, the sustenance that mediates the stimulus approaches the growing cells by means of an external source; this is the nutritive juice in the brood chamber of the mother. The higher or lower concentration of this stuff is the chief determinant of whether the young animal receives a high or low head. This juice is thought to exude from a cell layer on the floor of the brood chamber, where it itself is obtained from the nutritive pool of the mother's blood. Additionally, the yolk content of the embryo appears to contribute to helm formation in embryos and young animals.

In the second case, of the formation of the helm before the molt of the grown animal, it is the greater or less nutritive content of the blood plasma that acts as a regulating stimulus. Furthermore, the activity of amoeboid cells comes into play, which are found at the locus of helm development (and also at the base of the mantle spike) in the form of a small plaque. This is likely composed of 'blood cells'; their activity can tentatively be identified with the influence of the blood upon the helm or spike development. See Fig. 7.

Unfortunately, the processes of assimilation are researched little or not at all in these Entomostraca ${ }^{13}$ (see appendix 2, p. 28). One may however learn something of this process by means of the complete transparency of these animals. If given rich food, a heretofore malnourished Daphnia will very quickly begin to show characteristic alterations. The gut will immediately fill with algae, and one can then measure the time to appearance of the resorption droplets in the gut cells. Then follows a cumulative alteration of the heretofore invisible (totally water-clear) plasma into a visible juice, at first yellow and then gradually rather opaque. From this blood juice the whole body is provided with a rich stream of nutrition. In a surprisingly short time (at permissive temperatures), one now observes the previously invisible gonad swell, and the previously nearly fatless body swells, showing a characteristic red or violet color. The action of the enriched bloodstream on the helm-developing cells is similar: the cell layer increases through imbibition and perhaps also increased number of cells, and by the next molt the head height shows a substantial increase. Conversely, one can decrease the head-height through a molt by starvation; this has been described by Wesenberg and others. Even for some time before the molting, the new smaller helmet Anlage is embedded in the earlier one, such that the two contours are observably separated (Fig. 7).

We can finally state that, at a given temperature, each quantity of food corresponds to a specific increase of the head height, just as it corresponds to the buildup of fat bodies and ovaries. For each biotype, there are set rules for the allocation of available surplus (as absorbed and dissolved in the blood) to each different region or organ of the body, and to the increase of energy production (heart rate, swimming motion). For example, there is pronounced competition between the ovaries and the helm-building cells. In each case, the severity of the 'war' between ovary and helm depends upon assimilation (quality of food, temperature, etc.), on the special character of the biotype in question, and most importantly on the generation number.

In the 'generation number' we have a 'cyclical variable', which is to say a successively diminishing role of an innate factor in helm development. This inner factor, the 'helmpotential', reacts in different generations with different intensities to the same fixed environment. By this I mean nourishment and temperature and therefore assimilation, which we previously learned to be the regulating causes or regulating stimulus of helm development. Across many forms of Hyalodaphnia cucullata with similar nourishment etc., I noticed that the first generation reached a markedly smaller

\footnotetext{
${ }^{13}$ translator's note: an outdated taxonomic category of crustaceans.
} 


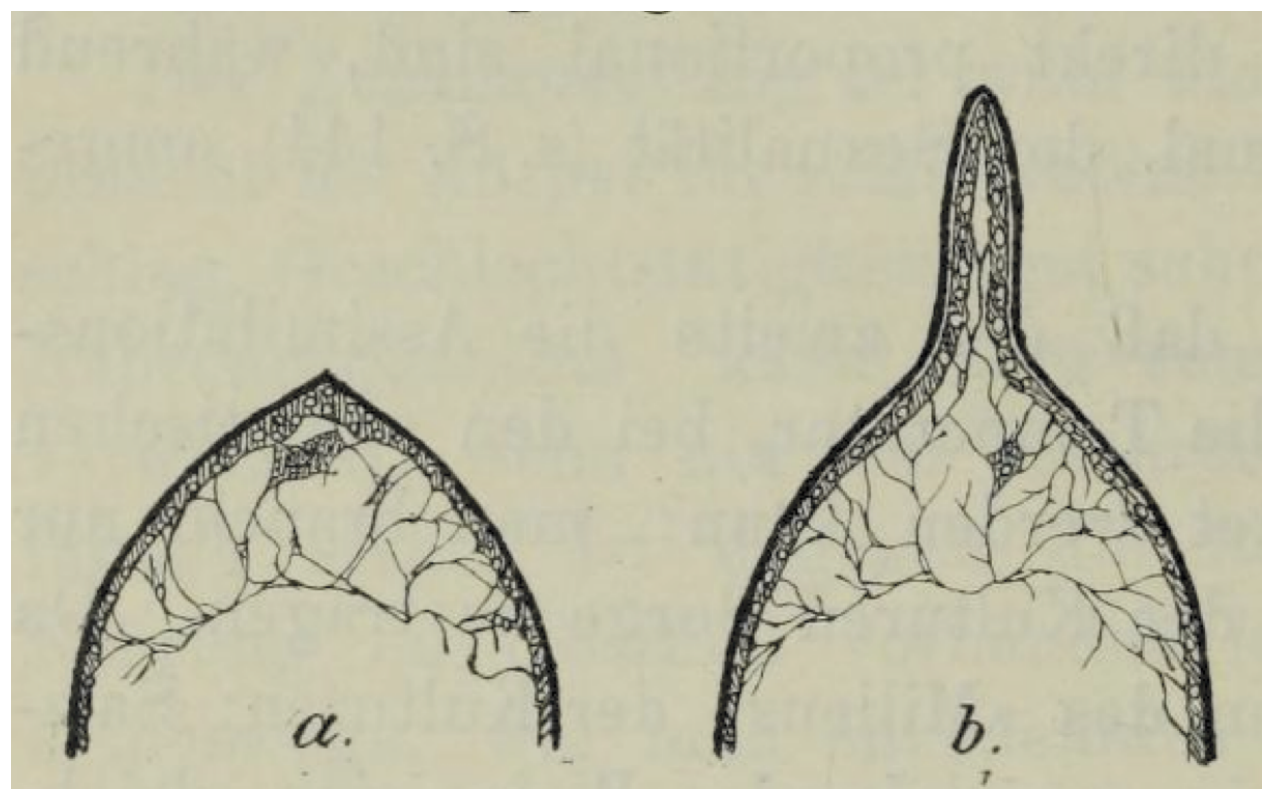

Fig. 6. Two frontal sections through the crown of a Hyalodaphnia, conducted approximately in the plane of the 'long axis'. Chitin in black, hypodermis cross-hatched with white inset kernels. (Underneath branched connective tissue single large amoeboid cells are shown.) $a$, Helmless animal (as Fig. 5 at left); $b$, Beginning helm growth.

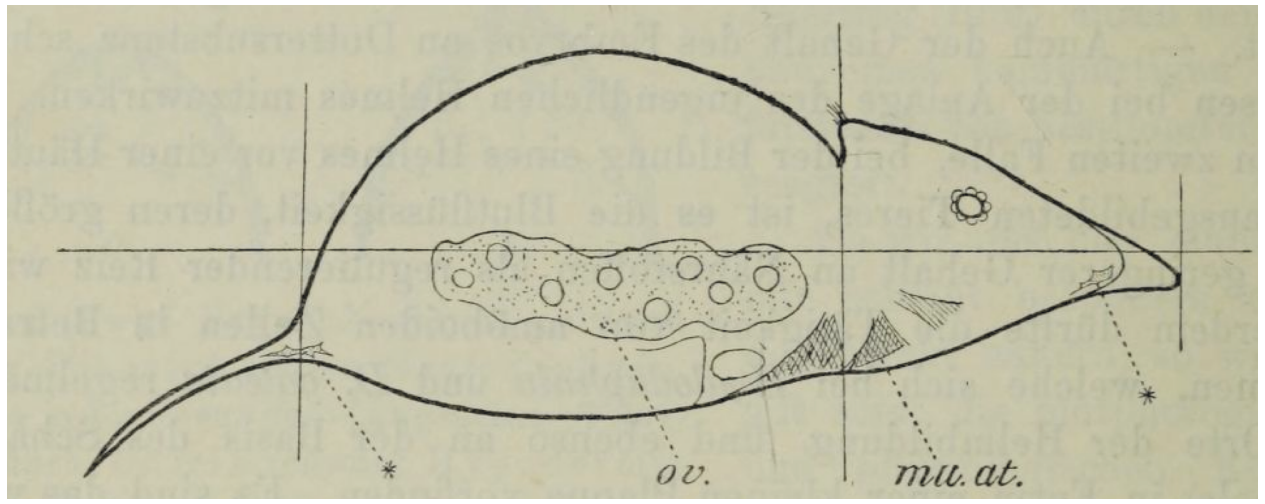

Fig. 7. Hyalodaphnia-9 shortly before molting, with diminished nutrition (or assimilation). The contour of the new helm is already visible, it is already become substantially diminished. * indicates plaques of amoeboid cells (blood cells?), which play a role in helm and spike development. ov ovary. (Compare also the explanation of Fig. 1.) 
average head-height compared to later generations. Further, for two local biotypes of the same species I can state that the middle generations reach a higher head-height than the later, in a similar environment.

Here it is worth noting that the early and late generations of these elementary types normally occur in the spring and in autumn, when in the wild they can develop only an inferior assimilation intensity relative to those in the summer. Thus other factors of assimilation are noticeable, in addition to the regulation of nourishment quantity by temperature.

With these, we have learned the innate factors of helm development: the innate cyclically diminishing helm-potential over generations; and on the other side the external factors: first nourishment, second temperature, and further the gas and salt content and other qualities of the water. From all these various external factors results the dramatic variability of assimilation intensity, to which we have learned to attribute the similarly variable head-height. Otherwise expressed: the assimilation intensity acts as a graded stimulus, that releases a corresponding intensity of reaction in the substrate of the helm-building cells. This reaction leads to specific heritable helm-potential in this same substrate.

\section{b. How do we determine the collected phenotypes of head-height for an elementary type, and how do we represent them?}

We desire to study the development, inheritance, and changing of these specific helm-potentials. From these studies, we desire to see whether the position occupied by De Vries and Johannsen - change of the quantitative trait only through mutation and determination by the environment - is supported or not. Through our knowledge of the helm-building factors, we are now in a position to make determinations regarding the causes (via induction or regulation) of all possible head-heights independent of each phenotype that this character depends on, for each elementary type that we investigate.

Without giving all the figures independently that were found for different biotypes, we wish to briefly consider how one may conduct an analysis of the collected phenotype ${ }^{14}$ of a character. Further, how may one best represent this analysis to lead to a solution of the question we have posed?

To simplify this general representation, we confine ourselves to the triggering cause (assimilation intensity) related to the factor of nutrition, and attempt to isolate the graded effects of this factor for study. We will leave aside the lesser causes of temperature, light, and water quality, by considering only cultures in which these factors are held constant and only the quantity of food is varied. (A study of such cultures in which nutrition is constant but other factors, such as temperature, are held constant, would not be novel. Rather, it would arrive at the same goal by an alternate path.)

Moreover, we observe only the effects on 우 and leave $\sigma^{x} \sigma^{x}$ alone. Finally, we restrict ourselves to only three levels

\footnotetext{
${ }^{14}$ The 'phenotypic aggregate' (Gesamtphänotypus) of a character we may unfortunately not say, because Johannsen has defined this term as the sum across characters of an organism, for instance their average in some particular condition.
}

of nutrition: poor, middling, and rich. Our task is to determine the phenotype of head-height for each such level.

To achieve precise nutritional quantities, we use carefully filtered water with certain quantities of Chlorella vulgaris (or similar algae such as Palmellacea, Kirchneriella lunaris, or others) added back from pure culture. We must transfer the animals daily to replenish algal quantities, because otherwise it will rapidly grow due to favorable conditions, and likewise, algae will be likewise rapidly consumed by Daphnia under unfavorable conditions. This transfer can be effected with a pipette, or for large cultures it may easily (and with practice speedily) be carried out with silk gauze. For "poor nutrition" conditions, one provides enough algae to sustain life, and also to allow reproduction; though only one or two eggs are desirable. The "rich nutrition" is attained by supplying algae in excess, and is immediately recognizable by the green color of the cultures. In this greening and the overabundance it signifies, one still finds impairment of some Daphnia (decrease of head-height and general vitality). Otherwise, in a culture made thick green-yellow with algae, Daphnia do well, and it is only here that the maximum head-height, body size, and speed of movement are found. (Curiously, one sees such natural forms in the animals that live in pelagic zones of clear lakes, for instance D. longispina of the Lunzer Untersee) ${ }^{15}$. "Middle nourishment" lies in between the empirically determined minimal and optimal quantities ("poor" and "rich"). The correct input of algae is requires some attention, and in judging it one considers also the quantity of Daphnia in culture. The method by which one may know the phenotype or mean value of a character for a certain life stage is well-known enough. One uses a large number of measured individuals to construct a frequency curve ("Galton curve") or, as is simpler in some cases, a distribution schema. From one or the other of these one reckons the mean value of the relevant character.

This mean value gives expression to the following input: the "normal" nourishment of the eggs and embryos in the mother animal ${ }^{16}$ for the collected individuals of the relevant lines in these cultures (which are essentially identical; see appendix 1), for some specific conditions of life. The higher variants owe their higher head above all to accidental chance, that an abnormally small number of eggs fully matured in the given environment. The lower variations owe their deviations similarly to too great egg production all at once. In both of these situations food quantity per animal is misstated. ${ }^{17}$ However, generally the egg production of agematched 웅 raised on similar environments are rather constant. The mean deviation (variability index) is here in most cases not meaningful, because in this case it gives large differences and complications even within the same Biotype. We therefore prefer to neglect this index. Figure 8 shown here represents no specific instance, but rather a schema for

\footnotetext{
${ }^{15}$ translator's note: Later work found this natural phenomenon to be due to predation. Woltereck's own later hypotheses on the subject were not borne out; See JL Brooks (1965) in PNAS.

${ }^{16}$ trans. note: note that in this case "normal" is not a synonym for "Gaussian".

${ }^{17}$ translator's note: I added this explanatory sentence.
} 
the head-height frequency curves that we obtain for Hyalodaphnia.

We can draw the three helm-height frequency curves for poor, middling, and rich nourishment in the same coordinate system (Fig. 8), and with them the three corresponding mean values $m 1, m 2, m 3$, the three phenotypes.

In overview, let us once more represent the dependency of the same phenotypes on the conditions of life (nourishment) through the following graphical representation. I hold on the abscissa axes the nourishment quantity, from zero on the left to overabundance on the right. As the assessed value, one may use either 1 cubic millimeter of an intensely green Chlorella nutrient solution or 1 small platinum loop full of Chlorella from an agar culture. Both quantities are agitated in a certain quantity of water (that differs between species). Mathematical exactitude in measuring this dose is obviously difficult, and unnecessary.

I draw points on the ordinate raised from the abscissa by a value representing the mean value of head-height derived from the frequency curve, to which the nourishment quantities correspond. ${ }^{18}$ When I connect the end-points on the ordinate, I obtain a curve (Fig. 9, 12) which I will describe as the "Phenotype curve". The curve constructed thus can be applied to some quality of the water (temperature etc.) that is shared between all cultures.

If I want to represent all the phenotypes of the analyzed character, I must next fill in all the relevant temperatures on the abscissa of the phenotype curve, or at the for low, middle, and high temperatures. Through this I would obtain three phenotype curves of the same character for each elementary type, the ratio of whose positions against one another will be somewhat different, as determined by heritably fixed differences between Biotypes.

Further, I must distinguish between the first, middle, and late generations (computed from dormancy onwards) for some elementary types. The form and the relative positional relationships of these curves are also heritably fixed for each Biotype.

Upon such analysis, the different generations of some Biotypes not only show different reactions to the provided food quantities, but also to temperature differences while holding food constant. It is therefore necessary to consider three temperature curves for each generation.

Finally, the curves for the helm-height of the ofo and the often quite different $\sigma^{\top} \sigma^{7}$ animals are distinct from each other. In one such case, the $\sigma^{\top} \sigma^{\top}$ animals of two elementary types differ, whereas the $q$ o animals show great similarity (the colonies from Frederiksborg and Moritzburg).

However, to attain a tolerably complete representation of the phenotype regularities, it would "suffice" for such a quantitative character as head-height to fill in the already not-insubstantial count of nine phenotype curves, namely for the control of the 우 in the following combinations: ${ }^{19}$

Beginning generations for low, middle, and high temperature,

\footnotetext{
${ }^{18}$ trans. note: In modern parlance, Woltereck has created a density plot. In his time, this was a less common procedure.

${ }^{19}$ The following orthography follows Woltereck.
}

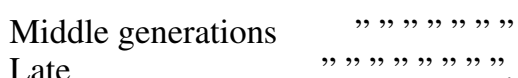

Now, each phenotype curve of the analyzed characters is based upon the mean values of at least three frequency curves, for poor, middle, and rich nourishment, so for approximate knowledge of the specific reactions of this single character we must use no fewer than $3 \times 9=27$ cultures and from them obtain frequency curves and their related middle values.

We must neglect to go through the details of all these numbers. In this instance, we can more simply clarify the consideration of quantitative characters as follows: the particular head-height of a given elementary type is heritably determined through a large number of relationships.

Even with these 27 related values, we remain quite far from an exhaustive definition of the specific helm-height and helm-potential of a tested elementary type. On the one hand we have left out all the intermediate values between the three chosen values for each of the helm-building factors. On the other hand, we have left out specific differences due to external factors, gas and salt content of the water etc., and also the effect of intrinsic regularities. Examples of such intrinsic regularities include the correlation between helm-height and germ cell proliferation and the development of head-height from molt to molt. We may also categorize other particularities of the apparently simple head-height and its heritable basis with these factors.

Now, overall we have very little reason to see head-height as especially complicated in the regularities of its relationship to environment relative to other bodily organs. A more exact analysis of each quantitative character may yield a similar richness. The analysis of "helm-potential" is merely more readily measurable to prosecute than the particular reaction intensities of the burgeoning of the ovaries or the fat bodies, the eyes, and so on. (At the least, we will later (p. 19) approach "sexuality" more closely, as an entirely different sort of character in its corresponding relations.)

There is little cause to expect a principally different nature of quantitative character potentials in other organisms. For example the length of beans in Johannsen's biotypes would be such a character. This measurement's potential (gene) would be found to be related to specific aspects of the environment, even if we manage to test the single influences of its circumstance (as in Daphnia). Regarding the qualitative traits (coloration etc.) whose specific differences appear much simpler to the casual eye than those described here, I will not venture anything. Both sides of the argument have already agreed that they cannot be principally different from the quantitative traits and potentials.

And so, what have we achieved so far?

The understanding that any such quantitative character in its wholeness can only be fully understood and characterized by a great horde of variables (i.e. the relationships of reaction intensities to trait variations, including stimulus intensity and trait variations from nutrient assimilation). The whole of these relations may be represented only through a number of data tables, or more comprehensively through the 'phenotype curves' that I have put forward for this purpose. If we want to realistically analyze the importance of these 


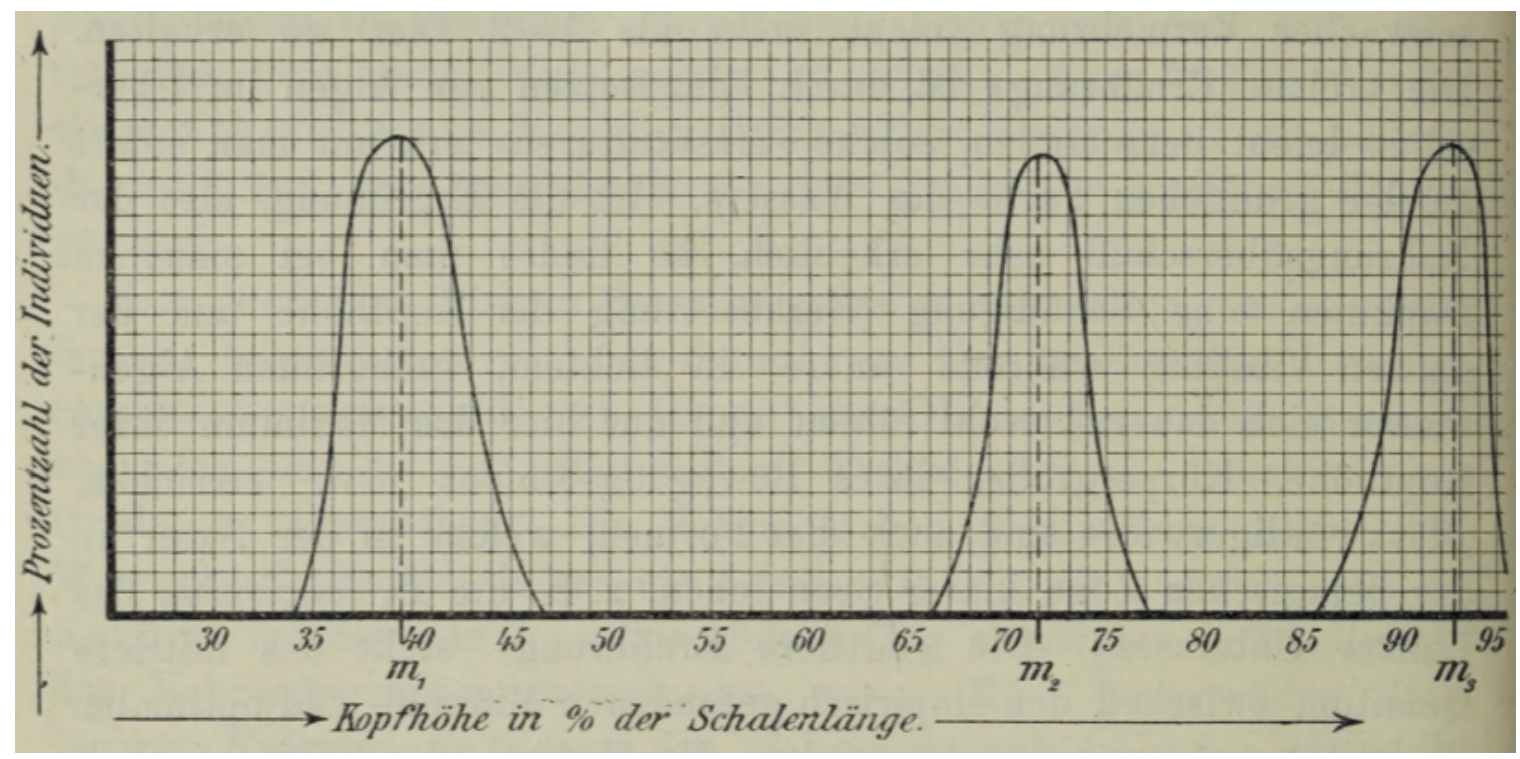

Fig. 8. Semi-schematic representation of the frequency curves for the relative head-height of a Hyalodaphnia-race, under minimal, middling, and optimal nourishment conditions, with the corresponding mean values: $m 1, m 2, m 3$. Abscissa label: Head-height as a percentage of the mantle length. Ordinate label: Percentage count of individuals.

various specific relations, the number of these curves is nine for our cursory evaluation here, and will grow to an endless quantity from there. The totality of these relations, described in these curves, we can call the reaction norm of the quantitative character in question. We can quiet this cacophony somewhat to the smaller number of phenotype curves that we actually have, which in praxi we must do. Many times over we will have to decide to construct a phenotype curve only for a "middle" generation and a "middle" temperature. We can then also from these curves speak of the "partial" reaction norm (Fig. 9) of the corresponding character. We may however go no further in our claims in these cases: we cannot take as given any measure of a quantitative character for which for which we have not at the least measured the same (in this case, middle) grade of nourishment (or assimilation).

\section{c. What can we infer from the genotypical basis of a specific head-height and the genotypical difference of this character for two elementary characters?}

The foregoing illuminates that we can define an elementary type and support it only through the specific and relative reaction norms of its different characters.

We interest ourselves in these facts not only because of their consequences for the systematics of plants and animals, but rather principally for their import for the sciences of heredity and speciation. To familiarize ourselves with this importance, we must recall two indubitable and eternal truths. First, the proposition that everything that is specific to an elementary type or a variety, by which I mean that which distinguishes it from other biotypes, must be bequeathed to the next generation in the germ cells. Second, a new type or elementary type arises through the heritable alteration of any specific character.
The "genotype" (the genotypical foundation) of a quantitative trait is its heritable reaction norm. From both of these propositions the following is very clear:

1. The total reaction norm with all its countless specific relations is inherited as the principle for helm development (helm-Anlage). Therefore, the "genotypical basis" or "genotype" or also the "gene" (all terms from Johannsen, the appropriateness of which we will not discuss here) for helm development are all equivalent to the reaction norm, as we have defined it.

2. The biotypes of Daphnia arise and have arisen through heritable changes in the reaction norms of their headheight and their other characters. From these reaction norms the natural question arises concerning our assumptions: are these reaction norms changed only through mutation, without any determining influence of the environment? Or are they continuously modified through the action of variable environments?

We can posit a discrete modification of helm development (if such actually exists apart from the more familiar continuous variation) only under similar conditions of the environment. Specifically, such a modification exists only if in similar food conditions the head-height appears by some quantity greater or lesser in one type. Such a difference transgresses the boundaries originating in embryonic nutrition that result in the continuous frequency curve of the phenotype. ${ }^{20}$ Such modifications can in fact occur; I have observed

\footnotetext{
${ }^{20}$ translator note: This position is more or less that of the mutationists and De Vries (see The Mutation Theory 1909, Vol. 1 Ch. 2 "Mutability and Individual Variation".): according to this view Gaussian curves arise for phenotypes due to environmental variations, whereas genotypic differences are "discrete" in that they fall
} 


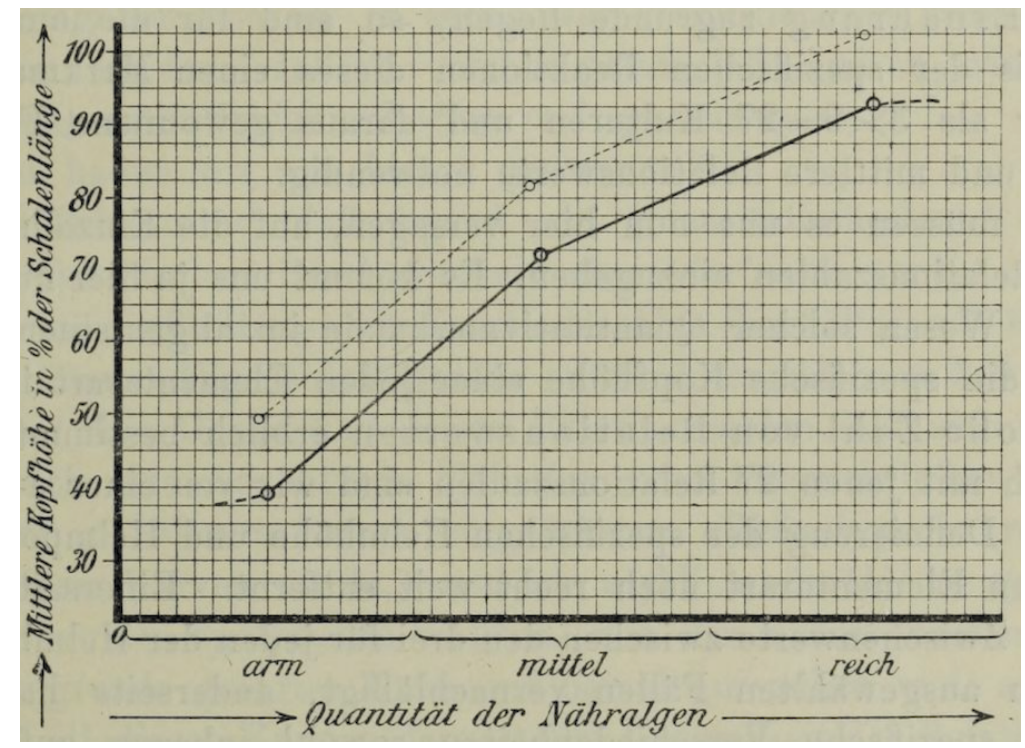

Fig. 9. The three mean values of Fig. 8 represented as the simplest "phenotype curve" (black lines). The animals represented belong to a middle generation, cultured in middle temperature.

them myself in the rostrum length of Daphnia longispina (Fig. 10). In all circumstances, such mutations naturally assert approximately the same difference of their mean from that of the original form. A comparison of the phenotype curves constructed for each such form corresponds somewhat to the picture in Fig. 11. (We can confine ourselves in the following to the reaction norms expressed in phenotype curves for a middle generation and a middle temperature.) For the case represented in this picture, both propositions of the mutation teaching are in effect:

The difference between biotypes is

1. discrete (I have also observed that half-races after the style of De Vries ${ }^{21}$ have heritable differences in rostrum length that do not cross over), and

2. not determined by the environment (because the difference develops equally at all grades of the environment, and can be attributed to no particular condition of the environment. Rather, the trait develops to its highest value through the joint action of both environment and the difference in the heritable stuff.)

These typical features of mutations can be dismissed no more in our Daphnia than in higher plants (De Vries and others), beetles (Tower), or vertebrates (Arenander and others).

The question is merely whether mutations can be ascribed a general and exclusive role in modification of types. And, as I show by means of the quantitative characters of Daphnia, this question can be with certainty answered "no".

First, remaining with helm-height, the phenotype curves of two elementary types (which through their various con-

\footnotetext{
outside the curve of their ancestor.

${ }^{21}$ translator note: Meaning a "race" that shows fairly discrete subpopulations even under true breeding.
}

ditions of growth become overlapping) must show a distinctive relationship such as shown in Fig. 11 if they are derived through mutation. There must therefore exist in each life condition a substantial and approximately constant difference between the two types.

This is how Johannsen understands his biotypes: they will show differences in phenotype in a common life history, which are the mutationally derived differences. These phenotypic differences reveal the differences between the genotypes of his elementary types. These genotypes are determined and stable quantities that must maintain the expected difference, as schematized in Fig. 11.

The beginnings of genotypical distinction: partially distinct reaction norms. - Natural Transitions. With my search for the possibly small but heritable differences between Hyalodaphnia landraces (and also after the beginnings of type divergence among such animals), I found also differences in reaction norms. That is, differences in the relative position of the phenotype curves which in no way fit the expected schema laid out above for mutations. Fig. 12 represents phenotypic curves of 3 local forms of Hyalodaphnia cucullata collected from middle generations raised at a middling temperature $\left(18-20^{\circ}\right)$. Under some conditions, the animals in question are so similar in their head-height that I had great difficulty distinguishing them from one another. This shows first that the three landraces are all nearly identical for the phenotype of head-height under low feeding (round, short heads). For middle sustenance a sharp difference appears.

One elementary type (from Moritzburg, appearing similar to the animals from the Trentsee ${ }^{22}$ and Frederiksborg lake)

\footnotetext{
${ }^{22}$ For this living material from the Trentsee I thank the generos-
} 


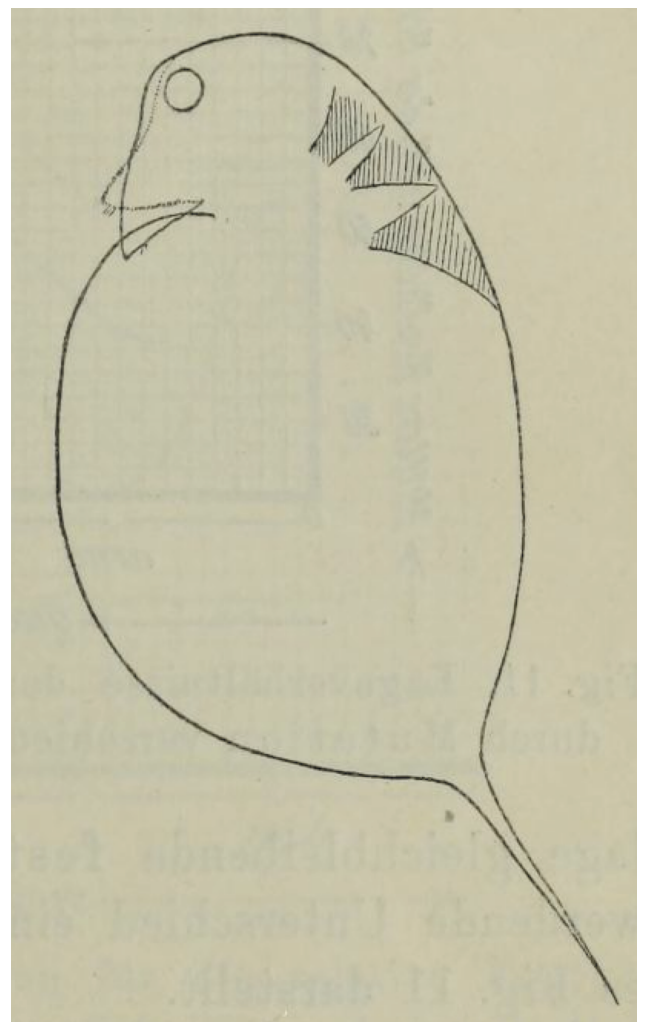

Fig. 10. A heritable, discrete recurrent variation of the rostrum length of Daphnia longispina (Lunz Untersee). The original contour of the rostrum is drawn in dotted lines.

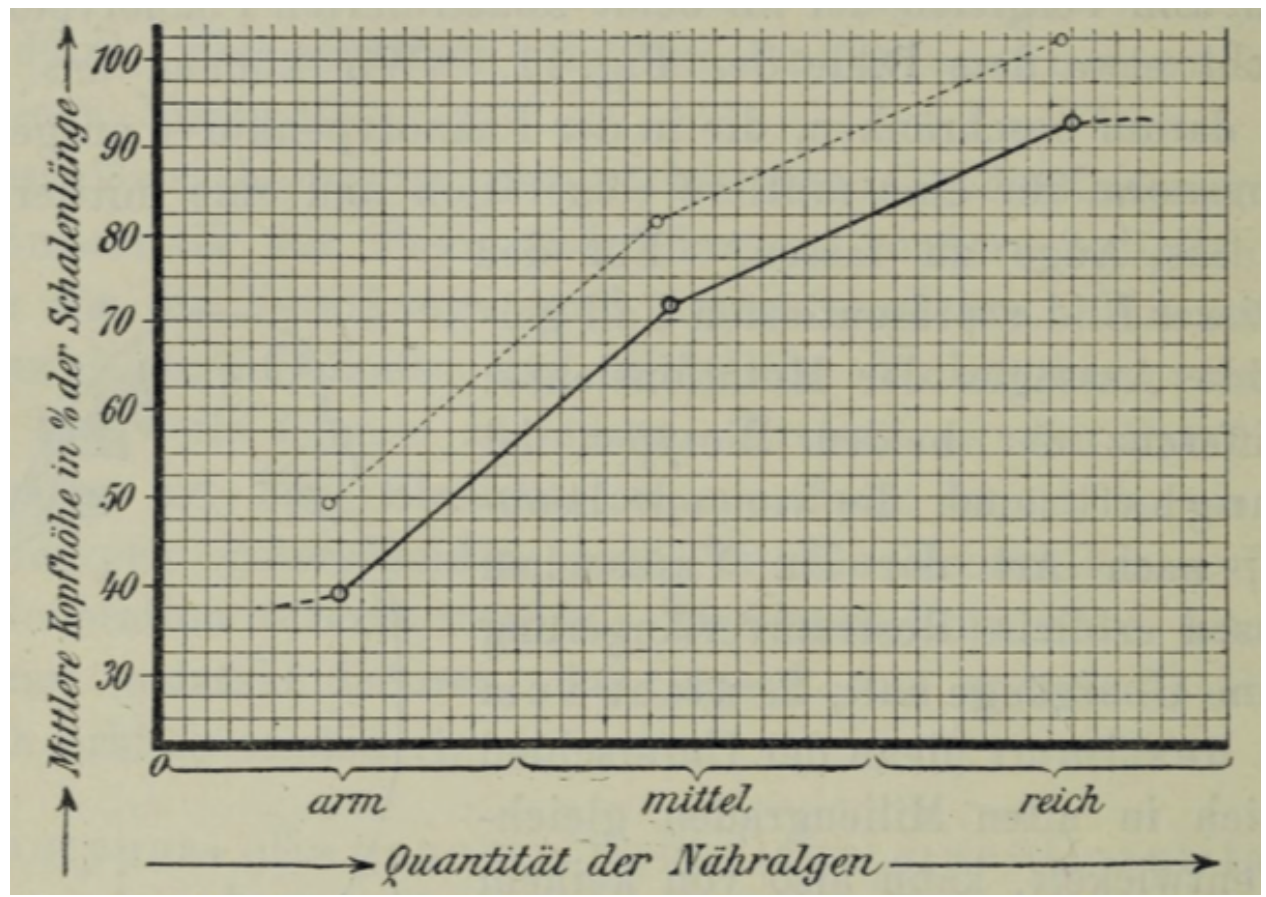

Fig. 11. A schematic: relations of phenotype curves of a character for two elementary types diverged through mutation. [translator note: note the strong resemblance to Fig. 9. It is not clear whether the similarity is intentional or an editorial mistake; in any case the figure is not obviously inappropriate in either case, and may simply constitute thriftiness of effort.] 


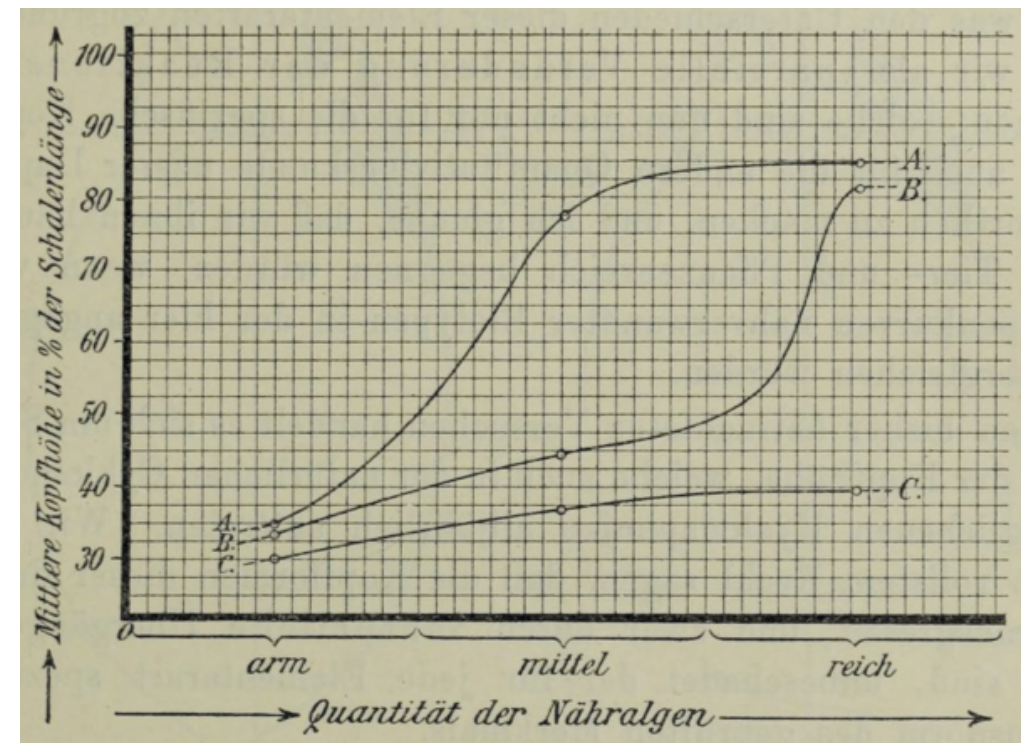

Fig. 12. Relations of the phenotype curvers for the relative head-height of three elementary types of Hyalodaphnia cucullata (우). A, Local type of the Moritzburg lake; $B$, the like from Borsdorf; $C$, the like from Kospuden. All curves show values for middle generations and constant middling temperatures.

already has a large head with middling nourishment, while with similar algal quantities the helm of the second elementary type (from Borsdorf) remains minimal and third (from Kospuden) has hardly any helm to speak of.

Further: the head of this last form is only slightly taller with rich food, while the Moritzburg and Borsdorf types develop very similarly tall helms with generous nourishment. These last two biotypes show similar forms at both lower and higher assimilation intensities, while they react quite differently to a middle intensity.

This behavior of the phenotype curves forms a characteristic contrast to the schematic curves presented for mutations (Fig. 11). To be sure, the quantitative differences between curves are genotypical and heritably determined, but this difference is even more certainly not abrupt and derived independently from the environment, because for each comparison it can confer large differences (for middle nourishment quantity) and also can completely erase a difference (as for worse and better conditions of nutrition). By the same token, the response cannot act through an invariant regular difference between two fixed genotype values, because the difference due to environment runs the whole spectrum from nil to full helm development. From this, there is no way to calculate a fixed value for a genotype across nourishment conditions. Finally, this difference must surely be caused by the environment factors (see appendix 3), as has been shown here through our precise control of the environment.

We can label the underlying differences between these elementary types as "partial alterations of the reaction norm". These are not specific to head-height, but can also be held accountable for other quantitative characters of our Daphnia. I believe that we will frequently encounter such alterations

ity of Prof. Zacharias (Plön). in the plant and animal kingdoms if we would compare the phenotype curves of closely related biotypes as I do here.

The experiments reported so far concerned variations in head-height, which also occur annually in the natural cyclomorphosis of these Hyalodaphnia. We can also say with the greatest certainty that the head forms of these three biotypes are transgressive, and overlap by means of "natural transitions". This tendency appears regardless of the character reaction norms specific to each elementary type.

The evidence that the Daphnia local forms are differentiated by means of continuous environmentally-determined alterations can lead to different conclusions. To understand it better, we next we consider more exact study of the transitional forms using manipulations in culture.

Artificial transitions agree with the natural relations of the environment. In my lecture of the previous year I showed head-height, spine length, and also "spine-angle" and "brow-angle", for two local elementary types of Daphnia longispina (Fig. 13). Indeed, these two types cannot be made equal by exchanging natural environmental conditions, except through a dramatic exaggeration of the differences in conditions. Specifically, the Untersee-Daphnia live in a naturally sparing environment and the Obersee-Daphnia live with substantially richer food. When I give the UnterseeDaphnia an overabundantly rich diet they become closer to the Obersee-form, and when I give the Obersee-form a very poor diet (which is to say that I starve them), they come to resemble the Untersee-form.

In this fashion a continuous series of transitions appears between the two elementary types with relation to a single character. These transitions show 1 . that the characters of the two types are not distinguished by discontinuous muta- 


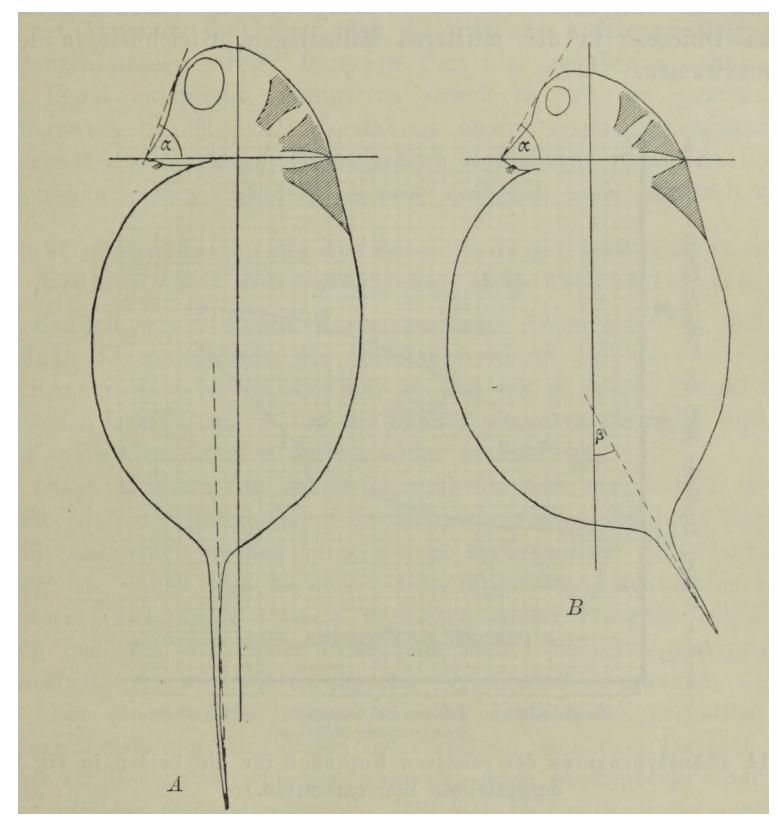

Fig. 13. Both elementary types of Daphnia longispina from the Obersee (A) and Untersee (B) of Lunz. (cf. the explanation of Fig. 2.)

tion, and 2. that these differences must be a function of the peculiarities of the environment.

Through persistently rich environmental conditions in the Obersee a natural "fat form" arises, and likewise through poor conditions in the Untersee we observe a natural "hunger form". Both forms are now heritably determined, such that under similar conditions their difference is fully preserved. This difference exists even under exchanges of conditions, as long as the conditions used are those that we observe in nature. That is, as long as we use the conditions of the sites from which these types originate. (Therefore these forms are in no way "site modifications", in that they already must be made alike by similarity of the environments. $)^{23}$

Only if we test the reaction of the character to extreme environmental insults (conditions not encountered in nature) do we find extensive similarity of the reaction intensities between the two types. Therefore when we compare the "phenotype curves", for example for head-height of the two elementary types, then we obtain a similar picture, like what we saw with the "natural" environmental curves $A$ and $B$ of Hyalodaphnia (Fig. 12): strong differences in the middling region of environment, but similarity in the environmental extremes.

To make the behavior of the aforementioned Daphnia longispina biotypes very clear, I have constructed the phenotype curves here (instead of the distribution curves I used in my publication of last year), because these frequency curves allow a better comparison for describing these Hyalodaphnia. I believe that the appropriateness of this step is obvious.

We may take this case as an example of such situations,

\footnotetext{
${ }^{23}$ trans. note: I am not familiar with the site modification terminology, potentially Woltereck means local adaptation.
}

that even if we cannot find "natural transitions" bringing types into phenotypic similarity, like the Daphnia of the curves $A$ and $B$ (Fig. 12), we can nonetheless identify "artificial transitions" through extreme conditions beyond the scope of natural environmental differences.

This last case we can label as the second step of biotype divergence (curves $A$ and $B$ would then be the first step). A third grade of divergence would be reached further in the cases represented by phenotype curves $A$ and $C$. Here the reaction norm of head-height is entirely different, while in the first two cases it is only partially different. We can hardly go wrong in the assumption that the temporal ordering of these divergence stages corresponds to the rules of this schema, at least for quantitative differences. As a preliminary stage of our first divergence class (curves $A$ and $B$ ) we observe a false site modification, whose phenotype curves remain identical in the conditions observed in nature.

Different reactions of successive generations agree with their natural environmental conditions. Finally, I ask the reader to recall that for certain elementary types, the reactions norms differ between generations, and thus we represent the phenotype curves for early, middle, and late generations differently in our analytical representation (p. 12).

This previous observation stands as further evidence that the potential for helm growth is not a unitary measure that can be altered by discontinuous single modifications. Also, in these generational differences there are even more dramatic transitions echoing the behavior of other biotypes, such that we do not require more data to recognize that the types are continuously rather than discretely differentiated.

However, we clearly see something different in the behavior of the successive generations. The first generation after 


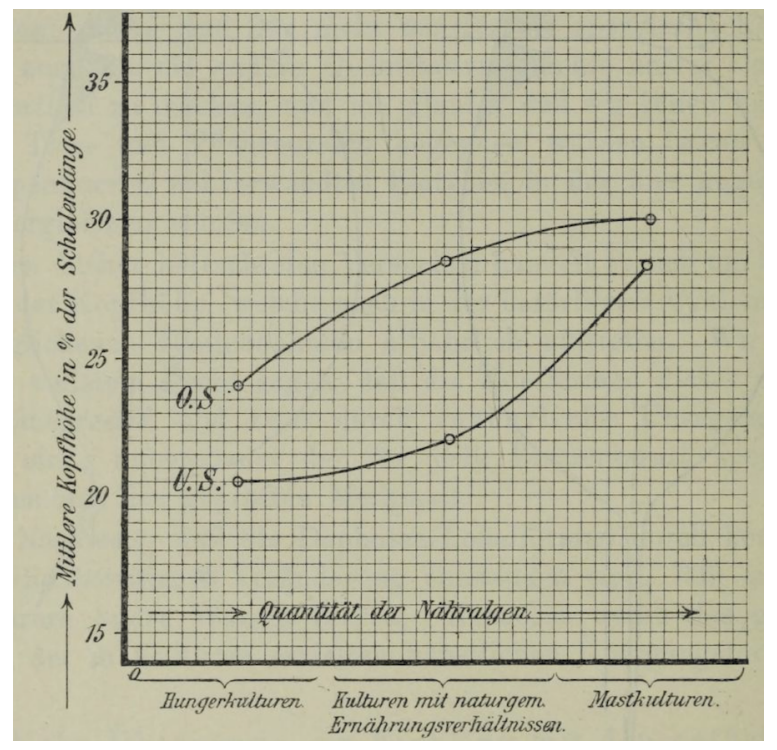

Fig. 14. Phenotype curves of the relative head-height for the two elementary types represented in Fig. 13.

dauer produces a smaller head than the middle generation under similar nourishment. We know this from the cultures, to which we can allow any quantity of nourishment we desire and simply supply the same amounts. It is difficult to find another explanation for this generational difference, except that the March-April-May generations ordinarily experience poorer nourishment in their waters (probably much poorer) than the later July-August-September-born animals. This predictably inferior nourishment of the first generations seems to likewise show its traces in the total potential of helm-height, as we see for the Untersee colonies. In both cases, this results in a heritably fixed diminution or hardening of helm-building in those generations (and likewise those landraces) in which helm-building has long been out of use.

In this interpretation we make the assumption that the higher heads in these special cases are ancestral. However, we can nearly take it as given that the richer nourishment of the summer generations and some colonies has gradually led the helm-building reaction to proceed more easily and with greater intensity in these generations and colonies. For our theoretical purposes these two statements are not materially different; the sleeping agency of a particular environmental condition has gradually and heritably fixed the corresponding grade of reaction (see Appendix 3).

Also, the late generations of a landrace that normally arise in the autumn show a decline in their helm-building ability compared to the middle generation not only in the field, but also in my cultures under similar conditions. These generations show a pattern of behavior that appears more complicated than the early generations, and but for the following line of thought, would require further explanation. I let it rest for the moment, and must only touch upon some points that are to me especially difficult and not quite settled. These regard the correlation between sexuality and head-height.

\section{Analysis of the phenotypic and genotypic differences of the quantitative character of sexuality.}

a. Exact determination and quantitative confirmation; causes of the differences We understand sexuality in this context as the tendency of an animal to have sexes ("strong sex") instead of being parthenogenetic ("degraded sex"). The sexual tendency expresses itself in the production of $\sigma^{7} o^{\lambda}$ or of mature $q 9+$ bearing ephippia (eggs requiring fertilization by males). We will briefly examine and compare each instantiation of the sexual tendency. These stand in contrast to the asexual tendency, with its production of feminine subitan eggs. (The production of subitan eggs does not on its own suffice to determine sex; these eggs can still produce $0^{7} 0^{7}$.)

The sexuality of any given animal (P) can be determined by examining its progeny, specifically by observing the degree of development of latent eggs ("winter eggs") in the ovary. For the most part it is sufficient to count young $0^{7} \sigma^{7}$ and latent eggs to measure sexuality, and on the other hand how many young 우 are derived by parthenogenesis. From this, we obtain a specific numerical value measuring sexuality. For example, "sexuality" $=100 \%$ (i.e. only males and Ephippia) or $=50 \%,=10 \%$ (i.e. half or $90 \%$ of the culture are parthenogenetically-derived $q$ \%).

Therefore, the sexuality of our Daphnia is a measurable and quantitatively determinable whole, just like head-height, spine length, egg production, movement and heart rate, phototaxis, etc. As I will show, this character is very similar to e.g. head-height in the influence upon it of the relations of nourishment, and thus the elementary types differ in a very analogous fashion in this character also. Indeed, we find that sexuality differences support our conclusions just as well as helm development does in the preceding section. Sexuality differences of the biotypes are also continuously distributed 
and determined by the environment.

We can prosecute our analysis of this trait in the same way that we treated "helm-height", and thus we treat first the slightest natural differences of type and natural transitions, then the "artificial" transitions, and finally the behavior of different generations.

Up to this point we aimed to determine phenotype curves for each elementary type, and thus to ascertain the degree of sexuality of each type under each environmental condition (of nourishment, temperature, etc.). However, for now we content ourselves with establishing that each elementary type has a distinct reaction norm for every character. In other words, we can determine the nutritional quantity required in culture (for a defined generation) to make (for example) 50\% of the culture parthenogenetic. We can establish that this quantity is different for each genetically fixed landrace. We obtain a specific phenotype curve for each elementary type, corresponding to the specific reaction norm of this character.

b. The beginnings of genotypical differences: natural transitions. When we search for the smallest heritable differences, and therefore the beginning of divergence, we also find forms for this character that do not differ at lower and higher degrees of some environmental parameter (with less and more nutrition), while they differ at middle levels. Such types show all possible natural transitions in their sexuality.

In the most informative comparisons, one picks out animals belonging to middle generations of two biotypes. (Earlier and later generations often show a less clear differences in reaction. The early generations are in very many cases purely asexual, whereas the later are in great measure sexual.) With these middle generations one finds that one type with middle nutrition shows $50 \%$ sexuality, the other shows $75 \%$, the third is already $100 \%$ sexual. All three biotypes are fully sexual when underfed, while with overfeeding all produce overwhelmingly parthenogenetic 우. The plotted curves correspond completely to those given for helm-height (Fig. 12). They are merely somewhat complicated by the stronger generational differences, and we shall therefore not examine them more closely in this report.

c. Artificial transitions, in agreement with natural environmental relationships. Likewise we find also for this character "divergences of the second degree" (p. 18), which is to say elementary types that are distinct under natural environmental conditions, but which can (for example) through overabundant nourishment make "artificial transitions".

I would like to bring up at least one example, representing an artificial transition between two extremely different cases. In some alpine pools near Lunz (at around 1600-1800m elevation) there lives a Daphnia pulex (obtusa), which exhibits a deeply imprinted cycle. These animals emerge from dauer eggs in approximately May/June to produce great quantities of parthenogenetic $\$ 9$, which then go over to pure sexual reproduction as early as August/September. While it is still to be established whether the stimulus for this transition is the reduction of nutrition, it is likely that the overpopulation of the pond itself would cause this. Then, in early fall, only ephippia and dauer eggs are observable. These will then rest for 8 months under ice and snow.

Now, I can treat these Daphnia consistently with their maximum assimilation (rich food and middling to high temperature), and in this way hold them over a year long purely parthenogenetic, without observing in these cultures a single $\sigma^{7}$ or ephippia. Through an unlucky mistake this culture was destroyed. My new cultures are now 8 months old and show completely identical behavior up to this point. (The consequences of these observations for the often-discussed sexuality problem do not belong in this article; see appendix 4.)

Through my cultures I can induce an artificial transition between the sexuality of this high mountain form and the acyclical reproduction of many lake cladocerans. Again, this transition indicates that such differences, at least when they are so large as in this case, cannot be derived by a discrete process. That they are environmentally determined is plain in this case. We can also establish, as with head-height (p. 16), that similar environmental factors (chronically rich nutrition) bring forth both acyclical colonies and our artificially acyclical cultures.

d. Reaction differences of generations, in agreement with the natural relations. Generational differences in sexuality are far more conspicuous but also rather more complicated than analogous differences in the reaction intensity of other quantitative characters, for example head-height ( $\mathrm{p}$. 18). These differences in sexual tendency are exhibited even under similar life histories.

Different forms of the cyclically fluctuating sexual reaction norm (specific "generational successions"). If we take a large number of Daphnia local forms from very different situations and cultivate them from ephippia, taking care to maintain similar conditions of the environment, we observe a somewhat different generational progression of sexual behavior for each elementary type. We can define a distinct "generational succession" of sexuality for each.

However, we can immediately obtain a tally of categories, which nevertheless are connected by all possible transitions and intermediates.

- First category:

(a) sexuality rapidly increases in the course of the initial generations (1-2-3) from $0 \%$ to $100 \%$ and is thereafter obligatory, which is to say that it solely dominates under all environmental relations from that point on.

(b) sexuality increases just as quickly, but it remains facultative and partial, which is to say that it can be satisfied with rich nutrition to produce some restricted parthenogenesis in varying degrees.

- Second category:

(a) Sexuality gradually increases over the course of 10 or more generations from $0 \%$ to $100 \%$ and at last becomes obligatory. 


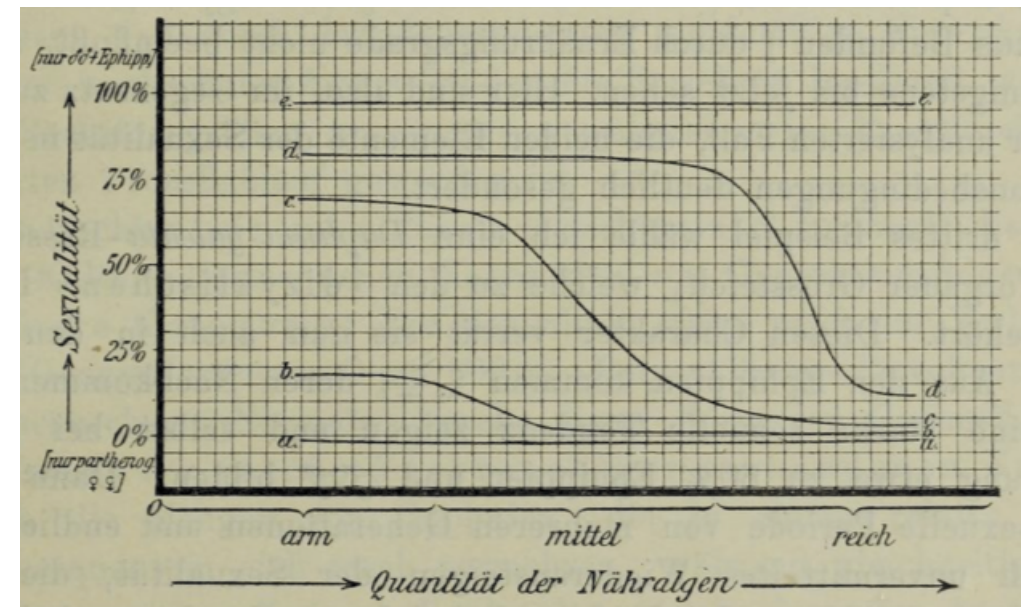

Fig. 15. Calculated phenotype-curves of sexuality for Hyalodaphnia (Borsdorf) for different generations. $a$, first generation; $b$, $c, d$, middling generations; $e$, later generations. Collected curves represented only for constant, middling temperature.

(b) The same, except that sexuality remains instead facultative and partial (in varying percentages).

- Third category:

(a) Sexuality increases very nearly as quickly as the first category, but never quite reaching total dominance. The progeny of the persistent parthenogens (or the ofo that can return to parthenogenesis) are almost completely asexual, but can regain sexuality gradually. After 6-12 generations these animals reach absolute and obligatory sexuality.

(b) The same, except that the second period of sexuality remains facultative and partial.

- Fourth category: All generations are obligatorily parthenogenetic; which is to say that even under poor assimilation they will not fall into sexuality. (We cannot begin these cultures from ephippia or from dauer.)

It would go far beyond our present purpose to study all of these categories in detail. What we wish to show, once again, is only this: sexuality is a quantitative character whose expression does not change discretely and suddenly. And second: this character's specific manifestations must be derived under the (direct or indirect) influence of the environmental relations.

Both of these conclusions proceed from the contingency displayed by this character, as brought forth by natural and artificial transitions. Both are illustrated by the different types of sexual generational successions, which surely derive their natural causes by these means.

We will now examine some typical cases from the above category IIa in Fig. 15, which are approximately the socalled "monocyclic" Daphnia. These races of Hyalodaphnia cucullata are found by Borsdorf.

The shifting of the reaction-curves or phenotype-curves from one generation is as follows. In the first generations obtained from dauer eggs, we find parthenogens at all levels of nutrition. The count of developed subitan eggs can be forced nearly back to zero through hunger, however not without the development of $\sigma^{\pi} \sigma^{7}$ and ephippia (dauer eggs) taking their place. After poor assimilation, sexuality appears in the following generations in ever-greater proportions, which is to say that a perpetually increasing nourishment is necessary to maintain parthenogenesis in the later generations. In the middle generations with middling nourishment we see sexuality of approximately $50 \%$, and only observe pure parthenogenesis with rich nourishment. Later, an ever-higher degree of assimilation is necessary to maintain even partial parthenogenesis, and likewise we finally observe absolute (obligatory) sexuality at all levels of the environment, just as we could establish absolutely asexual behavior at earlier generations.

We now consider the "polycyclic" pool form, as observed above (p. 19). These Daphnia belong to category 1a or $1 \mathrm{~b}$ characterized above. Under my direction V. Scharffenberg has experimented upon a race of Daphnia magna (that also Kerherv and Issakowitsch examined) from ephippia. He found that the tendency to develop fertilizable dauer eggs emerges very quickly, in the second and third generations. He established further that they arise also in the same $q$ as other eggs. In spite of this he could not fix obligatory sexual reproduction in the higher generations, unlike the Borsdorfer Hyalodaphnia and other Biotypes. V. Scharffenberg additionally made the observation that a Daphnia pulex o coming out of dauer stage is immediately able to produce a large quantity of parthenogenetic broods. These new parthenogenic offspring can directly thereafter produce dauer eggs in response to the stimulus of poor assimilation. (This also most likely holds for his $D$. magna race). The production of q 9 by these Daphnia-types (as discovered by $\mathrm{O}$. Kuttner [Freiburg] and V. Scharffenberg ${ }^{24}$ ) is not influenced by level of nourishment, at least so far as we can tell. In contrast to the case that I have analyzed, these reports examine the two elements of sexuality in clear separation.

As a third example, I choose a Daphnia galeata race from

\footnotetext{
${ }^{24}$ Both works appear together in the "Intern. Revue der ges. Hydrobiologie" etc.
} 
the large pond of Torgau, to which the "dicyclic" Daphnia belong. These characters betray themselves only in cultures. From ephippia come 우, whose descendants immediately show a strong sexual tendency and which themselves will show $60 \%$ ephippia and produce $\sigma^{7} \sigma^{7}$, given good nourishment. After this follows an asexual period of multiple generations and finally a rather abrupt re-establishment of sexuality. Finally, the sexual animals develop into a strong majority, around $80-90 \%$ given good nourishment. Obligatory sexuality is not reached, as in the the case of Fig. 15. In the course of further generations sexuality diminishes yet again.

These three examples serve as parerga ${ }^{25}$ to the table given on p. 20 of the different categories of sexual generational progressions, which suffice well to demonstrate one thing that I desire to show. Namely, the specific quantitative characters of our Daphnia are endlessly complex quantities, whose genotypic bases (the heritably fixed reaction norm) are linked by manifold transitions from biotype to biotype. It is impossible to consider these as discrete from one another among the volatile particularities of environment and generation.

The other thing that I wish to show, which all the discussed observations should demonstrate, is the environmental dependence of all quantitative traits.

\section{Relationships of the different types of specific "genera-} tional progressions" to the natural environmental conditions. The periodic (and sometimes inherited) fluctuations of the sexual reaction norm can only have arisen through the periodic fluctuations of those agencies that induce this sexual reaction.

Now, if we observe these periodic reaction fluctuations arising in our cultures while the intensity of those agencies remains constant, then it is hard to deny that certain changing reaction quantities have developed into a determinate process through a stiffening of the organism's reaction substrate. This occurs through longstanding and regular exposure to quantitative changes of these agent. ${ }^{26}$

From this point of view, we consider once more the categories of generational progression obtained from cultures:

First category of generational progressions: Sexuality rapidly increases in the course of generations 1-3 from $0 \%$ to $100 \%$ in these "polycyclic" denizens of small bodies of water. Normally, this form of generational progression is caused by the rapid depletion of the food supply through rapidly reproduction of Daphnia in a relatively small amount of water. The pond simultaneously shrinks rapidly from spring through summer due to evaporation. At the same time, the accumulation of azotic excreta (in the sense of Langhans, see his article in these proceedings) and the enrichment of carbon dioxide in the water through the Daphnia's increased respirateion (soaring numbers of Daphnia, and similarly, the depletion of algae suspended in the wa-

\footnotetext{
${ }^{25}$ translator's note: Greek, signifying an embellishment

${ }^{26}$ trans. note: Note again the clear idea of an evolved genetically determined reaction norm.
}

ter!) will work to curtail assimilation. This last is the actual causal stimulus for the increase of sexuality.

In this cycle-from dauer eggs through 1-3 parthenogenetic generations and back to dauer eggs-the substrate (gonadal primordia and germ plasm) in which this reaction process occurs is subjected to these conditions for very many generational cycles. Today, this process continues even without the habituated increase and decrease of the assimilation agents in many cases, in moderated form (facultative or partial sexuality in the later generations, or total sexuality, which is then maintained openly). ${ }^{27}$

For now we cannot be certain which (physiological) process is thus fixed. Some possibilities might be: assimilated energy from specific doses of the input food; warmth etc. in the gonads; the burden of the previously assimilated cell materials, which remain "normal" and therefore carry out normal sequences of reaction. Or perhaps (nearer to what we accept), the unaccustomed magnitude of those agencies correspondingly changes, leading to abnormal stimuli upon the substrate (germ stock). If so, then this substrate would be the actor persistently answering abnormal stimulus intensities with accustomed reaction processes. ${ }^{28}$

In each case the heritable stabilization of the specific generational progression establishes that we are in fact dealing with a genotypical character. Again, the obviously environmentally-dependent emergence of this character shows clearly that these genotypical characters and their differences among our elementary types are environmentally dependent (and not mutations). ${ }^{29}$

At the same time, we wish not to neglect another cause that may play a material part in the heritable fixation of the generational cycles, pending stringent and exact experimentation. This is the operation of natural selection (as rightly emphasized by Weismann), to which we can attribute the eminently suitable alternation between rapid parthenogenetic reproduction and the development of drought- and frost-resistant dauer eggs. Those individuals and populations that show less development of this alternation for are in point of fact threatened in their survival. Each freeze, famine, or drought leads to the decided extinction of all of its resident Daphnia colonies that have not developed dauer eggs.

One need not attribute all the peculiarities of the organism to the action of selection if they can emerge by simpler or more direct means. It would nonetheless be incorrect to refuse to consider natural selection when it so patently plays an enormous role in the phenomenon that we busy ourselves about. The appearance of parthenogenesis-so useful for rapid proliferation-from sexual reproduction is likewise attributable to selection. The same may be said of the fine structural design of the ephippia in their exquisite functionality.

\footnotetext{
${ }^{27}$ translator note: Here again Woltereck anticipates genetic assimilation!

${ }^{28}$ translator note: I have taken some liberties to try to make this paragraph easier to understand.

${ }^{29}$ Note that Woltereck argues not against genotypes, but against the mutational origin (in the sense of De Vries, not our modern sense) of the dramatic phenotypic variations he observes.
} 
In spite of this, we shall not at all concern ourselves here with the part played by selection in the emergence of Daphnia characters. Selection consists of an indirect action of the environment. This, together with the direct environmental effects that we have so extensively discussed, constitutes the "environmental dependence" which we desire to demonstrate for the characters of our elementary types.

Second category of generational progressions: Sexuality increases gradually in the course of numerous generations from $0 \%$ to $100 \%$. These colonies tend to be monocyclic Daphnia in larger bodies of water. The cycle ends either with obligatory sexuality or, with rich nourishment, parthenogenesis. Parthenogenesis may persist at lesser or greater rates, which can lead then to acyclic reproduction throughout.

Here the environmentally-dependent emergence of the progression is simpler and clearer to see than in the previous case, at least in the significance of its conclusion. The parthenogenetic generations correspond to the time of year, from spring-when the first $q$ hatches from the dauer eggs-to autumn-when assimilation is gradually reduced by the decline of both available food and temperature. This assimilation decline provides the stimulus initiating sexuality. This regular onset (e.g. in the 10th-11th generation) of the assimilation decline appears to be so solidly impressed in the gonad primordium and the germplasm that today we observe the sexual reaction emerge even without the seasonal variations of assimilation. If we talk about "qualities obtained by inheritance", we must be clear that such assimilation "qualities" (reaction intensities) and their alterations must concern not only the soma, but also the gonads and the germplasm therein.

Compared to the end of the cycle, it is more difficult to understand how the beginning of the cycle corresponds to the environment of the monocyclic forms. In many colonies, the first generations with obligate parthenogenesis experience less food and warmth than those generations later in summer. Nonetheless they are decidedly asexual at the beginning, and remain so with food shortages and cold, as V. Scharffenberg and I have shown with our cultures.

Other colonies begin their cycle first with richly available food and summery water temperatures. Have those earlyappearing Daphnia experienced an alteration of the sexual reaction through a secondary adaptation, where their hatching from dauer eggs occurs earlier and earlier? Or maybe in the home waters of these colonies in March, the "centrifuge plankton" that can pass through our finest nets are abundant, such as Lohmann has discovered in the sea? (I later found these also in fresh water as the most important food for the Entomostraca.) Or lastly, may there be a role for that baffling above-mentioned correlation between smaller headheight and parthenogenetic reproduction?

I will not discuss these possibilities here, or rather only indicate them, as they still await any exact test. At present it suffices to observe that the monocyclic reaction norm of sexuality must have originated from the action of the environment.
Third category of generational progressions: Sexuality initially grows nearly as fast as the second category, even with rich food. The parthenogens continue to deliver 우 descendants which are nearly all asexual, but gradually become more sexual. These are the dicyclic Daphnia, which end up with high rates of obligatory or facultative sexuality. The causes of this singular rhythm of the reaction norm are not yet explained. Two potential causes are currently under consideration:

First possibility: through the dramatic reproduction in the first generation, available food is quickly used up in the original small home ponds of this type, and assimilation follows. Sexuality emerges but remains partial, such that for a minority, straitened parthenogenetic reproduction is preserved. Then the population size declines precipitously, and the survivors find a newly enriched food supply (perhaps having to do with the manuring of the water with the innumerable carcasses of sexual animals). The population increases again until autumn, when assimilation drops again below category 2 . Through this we see the emergence of the second slowly waxing sexual period.

Second possibility: The first sexual period of the dicyclic Daphnia is a reminiscence of the short summer as it was after the last ice age. It was at this time that our current Daphnia were in part derived, and this long-ago summer length still acts to regulate these animals. The second sexual period is then a modern contrivance related to our current summer length. We do not need to discuss these two possibilities here (though the first seems more plausible to me), because in both cases it is the habit of the environment that underlies the constitution of the generational cycle. That alone is the important insight here.

Fourth category of generational progressions: Unconditional parthenogenesis under all environmental conditions that permit egg development. These are the acyclic Cladocerans of the large lakes and maybe those waters at warmer latitudes.

The environmental dependence of this extreme lifestyle lies plainly before our eyes. The acyclic races have lived for a very long time in waters affording them sufficient assimilation all year long, such that nearly facultative parthenogenesis (categories 1b, 2b or 3b, p. 20) can be maintained, which then in the course of time solidifies into obligatory parthenogenesis.

The races that have developed this complete sexlessness are the Daphnia longispina-hyalina, cucullata, and likewise the purely pelagic genus Bosmina.

Nonetheless, I bring up a colony of Daphnia pulex (which otherwise fosters polycyclic reproduction) from the warm lake of the Astroni crater in the Phlegraean fields. Since Spring 1906 this colony reproduced parthenogenetically without the appearance of a single $\sigma^{7}$ or ephippium when grown at $5^{\circ}$ under meager food conditions. They limit their egg production, and gradually cease egg production entirely and go extinct, but still show no trace of sexuality. At the time of collection, I observed a very few $\sigma^{x} \sigma^{x}$, so it is possible that this elementary type is just about to become fully 
asexual in the warm and food-rich Astroni lake. I immediately placed the animals in the Lunz station's hothouse with abundant food. Perhaps in my cultures this was merely the last prod towards complete asexuality. I return to this briefly (p. 27).

Therefore, we see that all the manifold and thoroughly "transgressive" forms of the Daphnia colonies lead back to the influence of the environment, as I believe they must. We can take from this phenomenon a second support that is at least as powerful for our conception of the alteration of types as that previously gained from the analysis of helm development.

\section{Investigations on the appearance of regressive variation and experiments on its production and fixation.}

These investigations involve two interesting characters of Hyalodaphnia: the nebenauge ${ }^{30}$ (accessory eye) and the "toothlet crowns" 31 of the young animals.

Both organs are normally missing from the local types that I investigated, and are rather endogenous features of the the genus' distant cousins Hyalodaphnia, for example Daphnia longispina-galeata. Their recurrence in Hyalodaphnia consequently represents a regressive variant.

I studied these regressive variants (and others) from this point of view: can at least these characters of my Hyalodaphnia, the absences of nebenauge and the juvenile toothlet crowns, be discretely (e.g. mutationally) derived? Both characters appeared to me to be discrete characters without transitional forms, and with no appreciable cause in the environment.

\section{a. The nebenauge of Hyalodaphnia.}

The genus of Hyalodaphnia (from Schoedler $1866^{32}$ ) is based on the absence of the nebenauge. However, in the intervening time Lilljeborg and others have acquainted us with a series of cases in which the nebenauge is also found in animals showing nearly all the characters of Hyalodaphnia (particularly the blunt rostrum with terminal scent antennae). Further, in some lakes such Hyalodaphnia with nebenauge occur in spring, whereas in summer typical Hyalodaphnia are found. It is therefore already very probable that Hyalodaphnia without nebenauge might be able to have offspring with this pigment spot.

We can compile similar results from E. Wagler (Leipzig) and my own cultures (especially the Frederiksborger Hyalodaphnia) showing that such alterations certainly appear, and in both directions.

I confirmed experimentally that in earlier generations (after dauer) the nebenauge is more common and more completely developed than in the later generations.

Already this contradicts my original conjecture, that we would like the character to suddenly disappear. The inherited

\footnotetext{
${ }^{30}$ translator note: the German term appears to be accepted generally for this feature.

${ }^{31}$ translator note: "Scheitelzähnchen"

${ }^{32}$ translator note: unclear citation
}

primordium of this structure shows the same periodicity that we became acquainted with in the characters of head-height and sexuality: some generations show the ancestral behavior, and only some the new, negative character.

With further investigation it was shown that we are not really dealing with a discrete character (absence or presence of a pigment spot), but rather all possible transitional forms between the original and the new behavior exist.

Indeed, we find transitions in two senses: either the cells of the nebenauge are more or less maintained but the pigment is missing, or the pigment is more or less developed while the cells are regressed. Each sign of the cell complex normally lying before the brain can be lost, and yet the characteristic black pigment granules are arranged, though only inside the brain matter (see Fig. 16, d).

Interestingly, the transitions concern not only the degree of development of these rudimentary organs, but also the level of heritability. I saw these pigment spots appear and then again disappear in different pure lines of the Frederiksborg Hyalodaphnia in a disordered, apparently capricious pattern. However, in some cases $q$ ㅇ had nebenauge that inherited these regressive variants to a rather high degree.

With one such $q$ that I isolated based on the welldeveloped nebenauge of its mother and grandmother, I achieved a nebenauge heritability above 90\%, which I was able to derive from the nebenauge-less Hyalodaphnia through only threefold selection and propagation of a Daphnia hyalina without nebenauge. In the $q$ with nebenauge, the regressive trait (the atavism) was so strongly impressed that some offspring with fully normal nebenauge (Fig 16,a) appeared. On all accounts, the organ remained very variable thereafter, and some nebenaugene-less individuals appeared in most clutches, even under continuous selection.

I am attempting to influence nebenauge formation through the removal of light and through extreme temperatures, but for now have not obtained clean results from these experiments.

We are therefore dealing with a quantitative (and regressive) character, that has no obvious relationship to environmental conditions. However, the manifold grades of nebenauge transitional forms can only appear through a continuously distributed degeneration of the character.

\section{d. The crown toothlet of the Hyalodaphnia.}

This inconspicuous organ is particularly notable, because its study revealed that the helm primordial cells and the tooth crownlet primordial cells are one and the same in Hyalodaphnia and Daphnia galeata. They are also found in many helmless races of Daphnia longispina. From this we can say with some certainty that the helm-building ability arises out of the older ability to generate the similar substrate of the chitinous toothlets. It is further found that transitions between typical "toothlets" and small superimposed head spikes occur.

It was shown through the investigations of E. Wagler (carried out at the Zoological Institute at Leipzig under my direction) that with many Daphnia (D. pulex, galeata, longispina, and Hyalodaphnia), all possible transitional 


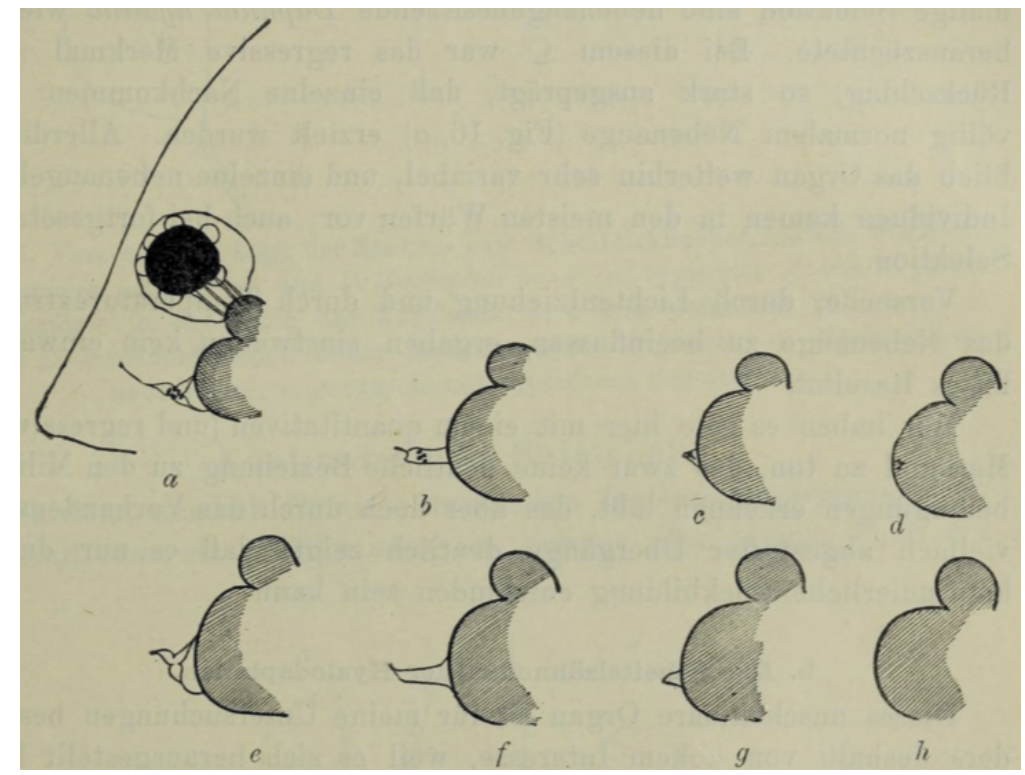

Fig. 16. Variations of the nebenauge in cultures of Hyalodaphnia (Frederiksborg). $a-d$, Pigment is contained, cells of the nebenauge in progressive deterioration; $e-g$, pigment is missing, the cells are however still present; $h$, cells and pigment grains missing.

forms occur between the familiar "neck toothlet" characteristic of so many Daphnia (particularly $\sigma^{7} \sigma^{x}$ ) and the typical crown toothlets of the young D. galeata and Hyalodaphnia. See Fig. 17 and 18.

We obviously do not wish here to further pursue a full history of helm development ${ }^{33}$, but rather only to bring into focus the appearance of the toothlets as regressive variants.

\section{Continuous emergence}

For many races of Daphnia longispina-galeata, the appearance of a toothlet on the crown of a young animal is a normal realization of an expected character. However, for the races of Hyalodaphnia that I am familiar with, this appearance is only observed in isolated cases, in which it is understood as a regressive variant.

We find manifold transitional forms for this character: 1. in degrees of development, 2. in prevalence of occurrence, 3 . in heritability, 4. in different strong tendencies for different generations. We have also noted certain complete parallels to previously observed regressive characters.

1. The toothlet can be longer or shorter, even unto merely a slight thickening of the chitin (Wagler). Further, it can vanish without a trace following the first molt, or the second, or the third as well.

2. A smaller or larger percentage of the young animals (from $0-100 \%$ !) may be furnished with this toothlet.

\footnotetext{
${ }^{33}$ I believe that once the neck and crown toothlets served in breaking the dauer membrane (as it becomes hard earlier than the chitinous shell of the young animal, and thereby made it possible to rip through the eggshell).-If this hypothesis is borne out, we may possibly have also an explanation for the uneven distribution of this character across the different generations. See p. 24.
}

3. Isolation of those young 우 with particularly welldeveloped and persistent toothlets in general does not enhance this character in the next generation (in relation to degree of development or to frequency of the character). Rather, the toothlet behaves like any phenotypic character. ${ }^{34}$ Nonetheless, in some cases I could demonstrate an increased frequency and therefore a greater heritability of the regressive variant, though still to a small degree than with the analogous nebenauge trait. While there I could achieve a nearly constant appearance of the old character (above 90\%) through only threefold selection, the crown tooth has not yet yielded a heritability above $50 \%$.

4. The repeatedly referenced Hyalodaphnia of the Frederiksborg castle lake, for which I thank the friendliness of Dr. Wesenberg-Lund, show a curious difference of this character across different generations. From the ephippia, which I collected in March 1908 (in Naples, where I had the ephippia-material shipped to me), thousands upon thousands of young Hyalodaphnia hatched. These nearly all possessed (around 95\%) a well-developed crown toothlet, that again receded in later generations. I believed then that I was simply dealing with an atavistic trait that was retained by sexually conceived animals but not by parthenogens, as for Nauplius larvae and the nebenauge of Leptodora ${ }^{35}$.

However, this explanation was contradicted by the observation that from the ephippia collected in March 1909 from similar origin, young Hyalodaphnia were born that

\footnotetext{
${ }^{34}$ translator note: Meaning here is not clear; possibly Woltereck is using 'phenotypic' to imply 'non-genotypic', or 'purely phenotypic'.

${ }^{35}$ trans. note: unclear citation
} 


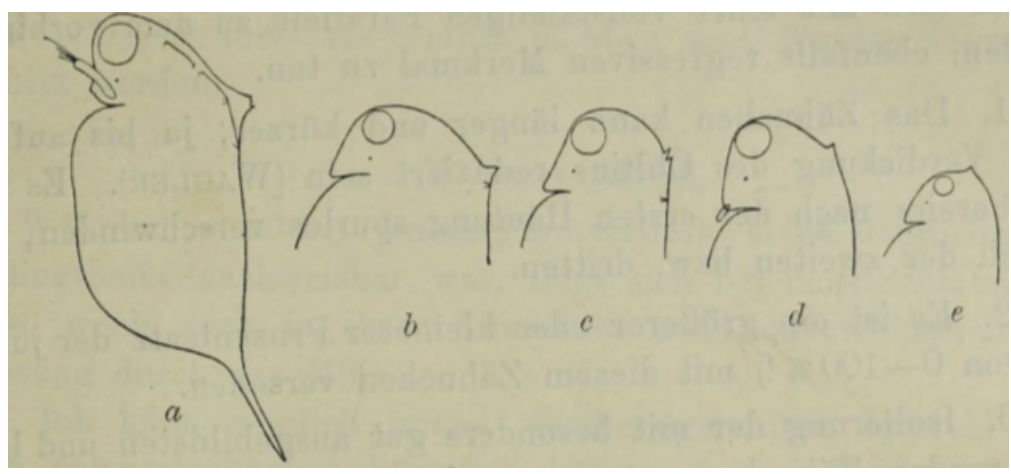

Fig. 17. Different locations of the toothlet between the neck and the crown after E. Wagler, for Daphnia pulex ( $a-b$ and Daphnia longispina (c-e. a, typical $\sigma^{\top}$ Daphnia pulex, with neck tooth in the line of the head base; $b$, $q$ with simple toothlet; $c$, young $q$, neck toothlet pushed up higher; $d$, young $o^{x}$, neck tooth yet higher; $e$, young $q$ with typical crown tooth.

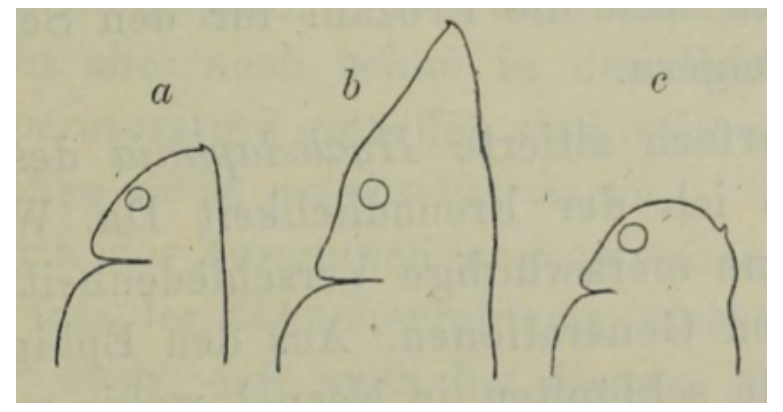

Fig. 18. Appearance of the crown toothlet for Hyalodaphnia (Frederiksborg). $a$, typical young form after hatching; $b$, the toothlet is retained in a fully developed $q ; c$, the toothlet appears again for an old $q$ as a secondary growth between crown and back.

possessed that toothlet at only $15-20 \%$ frequency.

There also, the grown descendants of these $q$ of only partially thinned out for this trait, such that the tendency of this development in the first generation is as heritable as in later generations; I can say indeed that it was similar to that calculated for the nebenauge above.

\section{Influence of the environment}

While there was no interpretable "environmentdependence" demonstrated for the pigment spot, an influence of the environment was apparent for the crown toothlet (also not yet understood in its causality).

Namely, I can artificially elicit parthenogeneticallyderived $q 9$ with crown toothlets by transferring mature $9+9$ from higher temperature $\left(25^{\circ} \mathrm{C}\right.$.) suddenly to a considerably lower temperature $\left(12^{\circ} \mathrm{C}\right.$.), and leave them there to produce young. In order to obtain this result, the eggs must not yet be present in the brood chamber.

From this outcome, which I was able to obtain in repeated cases, I drew the preliminary conclusion that the conspicuous difference between the young Hyalodaphnia of 1908 and 1909 ex ephippio may be explained by this observation. The dauer eggs in winter 1907/1908 were exposed to a reduced temperature at some critical developmental phase, whereas the dauer eggs of the next year experienced no such condition.
If the connection between this environmental agency and toothlet development is enigmatic for the moment, nonetheless we are confident that an environmental dependence is at play in this character.

We can now place these investigated regressive characters together with the previously considered quantitative traits of helm-height and sexuality. Nebenauge and crown teeth also arise in all their known specific grades of development by a continuous path. These organs are reduced and lost continuously, just as the possible degrees of development are continuous, and are in certain cases heritably fixed. For one of the two characters there is additionally definite dependence on environmental changes; for the other one this is not yet proven, but it is also not certainly disclaimed.

\section{Attempts toward the elicitation of new genotypical quantitative characters through extended action of certain grades of environment.}

Our observations so far chiefly concern the analysis of existing quantitative differences of elementary types. These differences were decomposed into their elements (the various phenotypes) in order that we might investigate their various causes. Then we could compare their overall differences (reaction norms), and draw some conclusions from their emer- 
gence.

Beside such analytical labors I have also busied myself about my Daphnia cultures, with the goal of heritably altering the reaction norms of my elementary types through longstanding exposure to certain grades of environment: in other words, bringing new genotypes into being. ${ }^{36}$

It will astonish no one that in the short time of three years only incipient results were achieved; however these beginnings are encouraging, and they agree well with the conclusions of the analyses we have already examined. So consider these here as suggestions of similar work likely to be communicated very soon.

I restrict myself therein to considering the main points of a series of assays regarding a single elementary type. These investigations concern the influence of extended hyperassimilation on the Daphnia longispina of the Lunz Untersee.

\section{Emergence of a genotypically novel degree of head-height.}

As I discovered in the work of previous years and have related above (s.141), this type is accustomed to relatively poor nutrition and low temperatures, but through rich nourishment and higher temperature it becomes very rapidly changed phenotypically through an increased assimilation intensity. Moreover, through such extreme environmental changes these Untersee animals become very similar to Obersee Daphnia in some (not all) characters. These characters are: body size, head-height, spine length, spine angle, and brow angle (see Fig. 2). As already indicated, in this form they are not identical, and therefore the Obersee Daphnia is not merely a local modification.

The assays that I describe now aim for the heritable fixation of one of these phenotypic changes (specifically headheight), and surely will not lead us to confer upon the Untersee Daphnia the appearance of the Obersee type. The latter likely represents the original biotype, judging from considerations beyond regressive variants. However, we can more or less induce a phenotypically taller head in each elementary type of Daphnia through overfeeding. Therefore, I would predict that this same modification will behave itself in a decidedly regressive fashion.

The present exercise is much more concerned with bringing from the character of head-height a genotypically novel value, and thus, heritably altering the reaction norm. Should this be achieved, it must therein be shown that our elementary type also reacts to its ancestral habituated environment (in this case limited food) with an altered head-height, and therefore has actually become a new elementary type.

Arranging the experiment is rather simple: one takes great care to maintain constant increased nourishment and temperature, and also that the number of individuals per cubic centimeter of water does not change too greatly. Furthermore, one applies constant controls on cultures; for example, through isolation of some $q \circ$ for fertilization. One then tests genotypical behavior from time to time (at a spacing of three to five generations), by isolating various animals into

\footnotetext{
${ }^{36}$ Trans. note: see again the identity between "genotype" and "reaction norm" in Woltereck's conception.
}

the original poor nutrition and determining the mean value of their offspring's head-height.

These tests revealed what I would like to share as a preliminary result, and thus cautiously: there is a noticeable shift of the reaction norm towards a genotypical stabilization of the head-height that was initially above-normal for the phenotype.

This shift has revealed three periods as yet in its "history", in which the cultures behaved appreciably different.

The first culture period. This period concerns the first generations after the placement of some 우 from the lake into culture glasses. In this new situation with rich Chlorella food and and the high temperature of the hothouse, these animals straightaway assume altered forms, which is particularly noticeable for head-height. This first culture period is a time of conspicuous variability for our Daphnia, in particular with regard to the head profile. The rostrum appears truncated in some animals, and in others hooked or beaked, and in yet others appears with a notch either near to the eyes or near to the rostrum tip. The brow angle (Fig. $2 a$ ) is consistently increased in these cases; the reduced and receding head uniformly shown by Daphnia from the lake does not appear in these cultures.

However, the multitude of head forms turns out to be a very ephemeral and purely phenotypical phenomenon; when placed with sparse food, similar to the natural conditions of the lake, the animals mostly build themselves the typical Untersee head by the next molt. And, most interesting to us here, the head-height changes to the original head-height in the time of a single molt after transfer to the old environment.

The second culture period. This period begins 3-4 months post captivity. It is characterized by development of a singular head-form with weakly concave brow contours. The aberrant profile forms appear more and more seldomly, and similarly deformities of the eye, the spine and mantle, hermaphroditism, and lastly the above-mentioned spontaneous variants (p. 11) become much rarer. All of these phenomena are characteristic of the beginning of the acclimatization, and also appear specifically when the cultures are contaminated by development of bacteria in addition to Chlorella or Kirchneriella, or through other influences. However, these same contaminants are less active in the second culture period, so one may readily speak of a continuous acclimatization to the environmental conditions (less room, rich food, high temperature). The Daphnia are now less delicate in their constitution, and similarly also less labile in their phenotype.

Most importantly, it appears that the animals do not reassume the earlier form so quickly upon replacement in the original environmental circumstances (poor food, low temperature). Younger 우우 (only 우우 were considered) need more molts to reach the original head-height phenotype, and older 우 cease to have so small a head as dictated by the level of nutrition. Nonetheless, the offspring born in the original environment of these same 우 have the original mean value of 
head-height from the start.

A sharp division of this second culture period from the first and third is of course not possible. However, I would like to have a step dividing the behavior of the offspring of the third period mentioned below and the behavior of the older 9 of of the first period.

The third culture period. This period has finally (June 1909) been reached by some of my cultures (from late summer 1907). Thus far, the offspring of high-headed females show a greater mean value of head-height even when they are raised with the original low nourishment and with low temperature. Indeed they may not only be born in the original environment, but their mothers may have young ovaries not yet divided into germ groups (egg +3 feeder cells) when they are removed from rich food and placed with poor food. In other words, these mothers first develop eggs in the original environment. Thereafter the hatched young animals had noticeably higher heads than we would expect from the original reaction norm of this elementary type for this degree of nourishment. In each case, an alteration of the reaction norm has occurred, or perhaps such a change is shown to us here at its first beginning in these transplants. Thus, we may be working with an alteration in the first generation raised under the old environmental conditions. The egg development leading to this generation occurred under the original poor nutritional relations, so we deal with more than a mere "after-effect" of the rich nourishment. A full sharp division of the terms "after-effect" and "gradual type-altering environmental effect" is unfeasible.

If one would prefer to interpret this reaction alteration as an "after-effect", it is nonetheless unmistakably a step from the merely phenotypic to the genotypic alteration of the head-height, and still agrees well with the greater part of the analytical results shared in this article.

Genotypical alteration of sexuality. As we have just subjected the "sexuality" of Daphnia to a thorough investigation, so here I would like to briefly indicate that this character too appears to be suitable for the study of the heritable influence of sustained environmental exposure. This is particularly true in such cases where there is already a tendency in the animals towards labile sexuality in one or another direction.

A pronounced tendency towards asexuality (acyclism) plainly exists in many races of cladocerans, especially for pelagic Daphnia and Bosmina.

Both Lunzsee Daphnia (Untersee and Obersee varieties) are in an apparently advanced stage of development in this direction. This seems to agree with my observation that each of these D. longispina cultures nearly completely loses the propensity for sexuality when left in the hothouse with rich food.

Moreover, in each case the later generations thus acclimated lose the ability to react (through sexuality) to minimal food and temperature.

Just so, the above-mentioned complete asexuality of my Astroni Daphnia (D. pulex) may lead back to a modification through the action of the environment. See p. 23.

And finally, the polycyclic Daphnia of the Alpine ponds appear to become sexual even when they are hindered by rich assimilation (p. 19), driving off the parthenogenetic tendency as if they were transplanted to meager food and temperature. Here also the original reaction norm shows itself to be altered.

Clearly all of these findings require a test of many years. Nonetheless, it seems to indicate to me that I should bring these Cladocerans to the attention of my colleagues, as they are apparently in a period of lability. The extraordinarily rich array of morphological and physiological characters gives witness to this lability.

\section{Appendices}

\section{The causes of continuous (fluctuating) variation in Johannsen's work and my own. (p. 9)}

${ }^{37}$ A crucial advantage of Daphnia over plants for our investigations is as follows: in each plant culture, as for example used by Johannsen, even under the most careful attempts to make experimental fields or patches alike, there are heritable ${ }^{38}$ differences in the environmental conditions of particular individuals. The same is true for fertilization and watering, the provision of room for growth, and further the available light for growing plants, and finally the quality of the soil that the sown seeds find for their rooting. All of these vary from individual to individual, and these are the principal causes for individual, fluctuating variation, as expressed in the frequency curve. The other causes rely on the quality of the sown grains, and thus (besides any differences in germ plasm) the mass of the nutritional material given to the embryo, in the thickness of the husk etc.

By contrast, with my Daphnia cultures the alikeness of the environmental conditions within the culture glass is absolute. If I fill such a glass with filtered water and load it with a dosed Chlorella quantum, each Daphnia living therein will experience completely alike conditions of existence. In there, they move unceasingly in the entire volume of water, and there is no difference from place to place. And there the suspended algae cells are filtered completely out of the water by the beat of their weir-like appendages, so the nutritional content of the water is even, and similarly the light and air concentrations, and the water quality.

The small individual differences observed as yet are therefore entirely attributable to bodily differences which the animals have from their birth. Therefore (barring differences of the heritable disposition), only differences in the nourishment of egg and embryo in the mother's body cause fluctuating variation in the Daphnia of such cultures.

And so, for us the conditions of the experiments are clearer and simpler than for the botanists. They must reckon

\footnotetext{
${ }^{37}$ translator's note: see De Vries' Mutation Theory for more background on the origin of the term 'fluctuating variation' and its relationship to mutationism and selectionism.

${ }^{38}$ translator's note: If this word is not an error on Woltereck's part, it likely refers to the opinion of De Vries that parental nutritional effects strongly influence progeny traits; i.e. 'erblich'/'heritable' was not synonymous with 'genotypic'.
} 
with two groups of individually varying and unknown quantities (embryo nourishment and individual position effects), and may never know how far their variants are attributable to one factor or the other. If they then go on to analyze the changing influence of the altered environment, they must hold always two different environmental influences separate: the general one that is "different throughout life history" (Johannsen, p. 219) ${ }^{39}$, and the individual one inside of a "given life history", which is to say in one of the so-far-as-possible alike culture patches.

Logically, Johannsen distinguishes between two types of environment-dependent variability, the collective or "group variability" that follows from general environmental differences, and the fluctuating variability (in the narrower sense) that follows from specific (individual) environmental differences. This necessary complication obviously does not reflect actual (objective) differences, but rather only methodological differences: for a given individual, it matters little whether it is affected by a special or general difference in the environment. Only the degree of our understanding differs. In the first case we know nothing about how far the individual form may be blamed on the environment (and which factors specifically); only in the second case, where the entire culture is changed in some certain way, do we know which factors of the environment we may thank for the general form of the individuals. But also in this case there are many individuals that in spite of an enriched life history (e.g. manuring) have in their own case met adversity (e.g. through crowding). Through all this the factor of "environmental influence" becomes something vague and unreliable in these experiments. It therefore becomes understandable why Johannsen dealt with it rather little in his fine experiments on selection in pure and mixed populations (and in these rare cases, never treated it numerically).

\section{Appendix 2: Towards metabolic physiology of the cladocerans (from p. 10).}

The volatility of the reactions of form paint an extraordinarily complicated but also especially appealing picture, demanding treatment with physiological methods. Unfortunately it has not been my luck to attract a physiologicalchemical collaborator to this problem, who would obviously have to develop their own specific methods for these small creatures. In any case it would be necessary to have gratuitous quantities of Daphnia for each single phase of the assimilation process to conduct a comparative study, such that each stage unfolding from famine to all different grades of assimilation intensity can be observed without resorting to microchemistry.

And if it should prove too difficult to attack conflicting assimilation processes individually, similarly, our understanding of overall metabolism in this organism is so poor that exacter investigation of the present phenomena concerning nutrition would be as welcome as of breathing. We can follow my methods of algae feeding to perform quantitative and qualitative analysis, taking also into account the metabolic

\footnotetext{
${ }^{39}$ translator's note: presumably Elemente der exakten erblichkeitslehre cited above.
}

byproducts in culture medium to make chemical processing possible. Of course, the solid, dissolved, and gaseous materials must be simultaneously investigated because, for example, the oxygen and carbon dioxide in the water will be dependent upon one another due to excretory matter.

Dr. Langhans (see his submission to these proceedings) has presented fascinating work concerning how the accumulation of metabolic byproducts damages the vitality of Daphnia. This often appears in my cultures, though very commonly there is no harm whatsoever, as in some cases when ("acclimated") animals were kept living in a small tube in great numbers for over a year. I believe that differences in water $\mathrm{O}$ and $\mathrm{CO}_{2}$ content sometimes yields a harmful effect, and sometimes not. Daphnia magna appear to be particularly resistant to accumulation of excretion materials in the form of $\mathrm{O}$ depletion and $\mathrm{CO}_{2}$ excess, but only under quite specific circumstances, which we do not yet know entirely. In the hothouse in Lunz I have repeatedly obtained cultures of D. magna in which not only nearly but actually developed a solid mass of 우 Daphnia, such that the animals could not move freely. Each was completely wedged in between the others. (This state of affairs occurred partially through reproduction, and partially because I allowed the water to evaporate.) There was in this water no trace of vegetation and the animals fed on stirred-up particles of bacteria-rich sludge that covered the bottom of the container. These cultures converted in part to sexual reproduction, though for weeks they remained in part weakly (1-2 eggs) parthenogenetic. I noticed no sign of degeneration.

Where did these animals obtain the necessary oxygen (at water temperature $\approx 20^{\circ}$ !) and how did they deal with carbon dioxide and excreta?? In general D. magna is rather sensitive to water deterioration. Freshly captured animals placed in great number in a glass tube often suddenly go extinct in hours, evidently due to $\mathrm{O}$-starvation and $\mathrm{CO}_{2}$ poisoning; Knorrich ${ }^{40}$ and Langhans (as shared in friendly correspondence) have also repeatedly observed that such Daphnia suddenly die out during thunderstorms. The chemical balance of "acclimated" animals must be completely altered. Hopefully the presentation of Langhans' interesting researches on excreta will give us a physiological view regarding the question of metabolism. And hopefully through my correspondence and his, further collaborators (specifically chemical) can be found.

\section{Appendix 3: Possible modes for partial alterations of the reaction norm. p. 16}

${ }^{41}$ Following my experiments regarding the perpetual exposure to some environmental condition, I presented the emergence of such "partial alterations of the reaction norm" as follows. Through the longstanding action of a certain assimilation intensity on the helm-building cells and the "potential" therein, one of the possible reaction intensities is continuously realized. This reaction process and its resulta certain head-height-thereby become "accustomed" over

\footnotetext{
${ }^{40}$ Plöner Forschungsberichte 1902

${ }^{41}$ translator's note: This appendix in particular is a lucid description of genetic assimilation.
} 
the course of generations, while the other possible reaction processes (=head-heights) become even so more "unaccustomed" as they perpetually fail to be realized. We can now suggest how the "unaccustomed" reaction intensities (= head-heights) gradually become more difficult to achieve, while the "accustomed", which up until now was only achieved via stimuli, becomes slightly exaggerated relative to the level of stimulus. Thereafter in such cases, an especially high head would appear only with higher grades of assimilation intensity than were previously necessary, and especially small heads would eventually only be achieved with yet poorer nutrition than was previously required.

One can then again also suppose that the "accustomed" reaction process, which corresponds to the ordinary amount of food and the various environmental relations of the pertinent locale, will in the course of time tend to appear more readily than the unaccustomed degree of reaction. (Just as an endlessly repeated flow of logic becomes more "fluent" to us than arguments we seldom encounter.) Such an "easy path" reaction will then succeed in the presence of stimuli weaker than the stimuli originally required to develop the same helm-height and helm form which is now habitual. This accustomed form will now appear with somewhat smaller (or on the other hand with somewhat larger) food quantities than were originally efficacious. Thus the alteration in the "accustomed" reaction process can be combined with the previously observed alterations in the "unaccustomed" reactions to result in a progressive fixation of the helm development adapted to a certain environment, such that it appears also in other environments. In the course of such a fixation process, the phenotype curve begins to take on the form of curve "C" in Fig. 15 (fixation of the lesser head-form following undernourishment) or curve " $\mathrm{A}$ " (advanced fixation of the higher head-form following lasting rich nutrition).

\section{Appendix 4: Sexuality and generational cycles.}

For the causes of sexual differences and sexual cycles of Daphnia, a problem much discussed in recent times, the collected experiments presented here allow some notunimportant conclusions to be reached.

I say in advance for context that today two camps stand against each other in these matters. On one side, the pupils of Richard Hertwig: Issakowitsch, Popoff, and others with the view that the sexuality of Daphnia is normally the aftermath of nucleoplasm alterations in the egg cell brought on by sustained parthenogenesis, or a similarly derived decay through starvation afflicting the nucleoplasmic relations. Hence the development of dauer eggs after long parthenogenesis, just as in cultures exposed to hunger and cold.

On the other side stand Keilhack, Strohl, Kuttner, and others, who follow August Weismann to show that cycles are inner, inherited, and arise through acculturation.

An exhaustive critique of the two views and their proofs is not my goal here; I will only briefly state what conclusions my cultures have yielded.

To wit, they evince the ability to produce parthenogenetic o eggs just as they are able to develop a helm or a spine or a colored pigmentation. In brief, parthenogenesis behaves like any morphological or physiological quantitative character.

Like the others, this character has twofold causes for its specific manifestation: external environmental factors and inner, heritable specific potential. As with the unfolding of each character, we see here that sometimes the outer factors dominate, and sometimes the inner.

For one Hyalodaphnia (from Borsdorf) I cannot up to this point remove a sexual tendency by any superlative conditions of life; here the heritable factors have triumphed.

For Daphnia obtusa (from Lunz), on the other hand, it is rather easy to maintain pure parthenogenesis at will through control of external conditions; here the external factors triumph.

We know yet more forms occupying all positions besides these two, including some that maintain parthenogenetic reproduction under adverse conditions. Here again, the influence of the environment is near zero, in these cases of continuous parthenogenesis. The inner tendency alone decides.

Now, which is the more important and characteristic of Cladoceran sexuality? That the phenotype of this character is very often substantially changed by the environment (Issakowitsch)? Or that in the development of this character an inner, cyclical periodicity emerges, that is rather genotypically defined and often sets itself agains all external influences (Weismann)?

Surely this last observation, and therefore Weismann's heritable generational cycles, lays bare before us the nature of cladoceran reproduction, while the dependence of the phenotype on the environment likewise shows a truth. However, this truth is so general for quantitative characters that we have brought in the term "reaction norm", to describe what the genotype actually is to these characters.

On the other hand, the discovery of those inner periodicities loses no importance if we additionally find in these Daphnia something similar for other quantitative characters.

The important point is rather that the inner heritable cycle can be experimentally proven, now that V. Scharffenberg and I have found regular strengthening in sexual tendency even with similarly maintained environmental conditions in our cultures. The same is true for additional specific forms that agree well with the naturally occurring mono-, di-, and polycyclic reproductive modes.

In understanding the mechanism of this sexual strengthening (and weakening, for dicyclism), we can hopefully still make good use of nucleoplasmic changes (when we will have studied these alterations for different forms of cladoceran reproduction).

At this point we know the following: the cytological (ideally visible) mechanism of periodicity actually has a basis in rhythms of the germplasm: the heritably fixed fluctuation of the reaction norm from one generation to the next.

Therefore: there are indeed generational cycles in Weismann's sense. 


\section{Discussion}

\section{Mr. Ziegler (Jena):}

Woltereck's investigations represent a fine analysis of types and make possible a thorough distinction between nonheritable modifications and heritable predisposition that has until now not been possible in the area of zoology. Researches such as those of Johannsen and the present author undertake to give the science of inheritance a new and stabler foundation. Certainly the science of selection is not thereby altered, because these authors busy themselves only with the assortment of existing races and subspecies, what I in years past once expressed in the following words: Nihil est in selectione, quod non fuerit in variatione. However the subspecies or local forms react differently to external influences, thus will they be imperiled by extinction to different degrees, if the battle for existence stages difficult challenges against the performance of the organism. The teaching of selection will therefore not be shaken by the new results from research in inheritance.

\section{Mr. Simroth (Leipzig)}

points out that the basic paradigm of mutation, represented by the new elementary types found in degenerate families of Oenothera lamarckiana in the pedigrees dealt with by De Vries, gives a clear articulation of the influence of the environment. Namely, all of the new forms appear or wax in the years 1894-96, and then again recede in a very conspicuous fashion. That implicates the maximum of the last cycle of sunspots, by which an analysis of the environment-meteoric and electrical influences-would be greatly advanced. Unfortunately, I do not know whether the statistics were compiled in the last sunspot cycle. I would like to direct attention again to the possibility that the propensity of Oenothera lamarckiana towards mutations is related to a reversion of the American plants under the influence of this oscillation. ${ }^{42}$

\section{Mr. A. Lange (Zuerich).}

\section{Mr. Woltereck.}

\section{Acknowledgments}

The digital version of the original article was digitized and hosted by the Harvard Biodiversity Heritage Library. I am grateful to AAAI and various people for implementing the AAAI style available on Overleaf used for this translation, and the www.dict.cc online dictionary as a general translation resource.

\footnotetext{
${ }^{42}$ Trans. note: I have no idea what this guy is talking about.
} 